\title{
Matrix factorizations and link homology II
}

\author{
MiKHAIL KHOVANOV \\ LEV ROZANSKY
}

\begin{abstract}
To a presentation of an oriented link as the closure of a braid we assign a complex of bigraded vector spaces. The Euler characteristic of this complex (and of its triplygraded cohomology groups) is the HOMFLYPT polynomial of the link. We show that the dimension of each cohomology group is a link invariant.
\end{abstract}

57M27; 18G99

\section{Introduction}

In the paper [8] we constructed, for each $n>0$, a bigraded cohomology theory of links in $\mathbb{R}^{3}$ whose Euler characteristic is a certain one-variable specialization $\left(q^{n}, q\right)$ of the HOMFLYPT polynomial (Freyd et al [4], Przytycki-Traczyk[11]). The $n=0$ specialization is the Alexander polynomial, equal to the Euler characteristic of the knot homology theory discovered by Ozsváth, Rasmussen and Szabó [10; 12]. The approach in [8] fails for $n=0$, assigning trivial groups to any link.

In this sequel to [8] we assume that the reader is familiar with that paper. Recall that our construction of link cohomology was based on matrix factorizations with potentials being sums and differences of $x^{n+1}$, for various $x$. When $n=0$, the category of matrix factorizations (up to chain homotopies) with the potential $\sum \pm x_{i}$ is trivial. Looking for a remedy, let us add a formal variable $a$ and change the potential from $x$ to $a x$. We will show that this potential yields a categorification of the HOMFLYPT polynomial. Namely, following the method of [8], to a braid closure diagram $D$ of an oriented link $L$ we will associate a categorification complex $\widetilde{C H}(D)$ of bigraded $\mathbb{Q}$-vector spaces

$$
\widetilde{C H}=\left(\cdots \stackrel{\partial}{\longrightarrow} \widetilde{C H}^{j}(D) \stackrel{\partial}{\longrightarrow} \widetilde{C H}^{j+1}(D) \stackrel{\partial}{\longrightarrow} \cdots\right),
$$

where

$$
\widetilde{C H}^{j}(D)=\bigoplus_{k, l} \widetilde{C H}_{k, l}^{j}(D) .
$$


This association has two properties: if two diagrams $D_{1}$ and $D_{2}$ represent the same link, then their complexes are homotopy equivalent

$$
\widetilde{C H}\left(D_{1}\right) \simeq \widetilde{C H}\left(D_{2}\right),
$$

and the graded Euler characteristic of the complex

$$
\langle D\rangle \stackrel{\text { def }}{=} \sum_{j, k, l}(-1)^{j+k} t^{2 k} q^{k+l} \operatorname{dim}_{\mathbb{Q}} \widetilde{C H}_{k, l}^{j}(D)
$$

is related to the HOMFLYPT function $F(D)$ of the link represented by $D$ :

$$
\langle D\rangle=\frac{F(D)}{1-t^{2}} .
$$

Here we define the HOMFLYPT function of an oriented link up to a normalization by the skein relation

$$
t F\left(L_{+}\right)-t^{-1} F\left(L_{-}\right)=-\left(q-q^{-1}\right) F\left(L_{\|}\right)
$$

where $L_{+}, L_{-}$and $L_{\|}$are three links which coincide everywhere except at one point where $L_{+}$has a positive crossing, $L_{-}$has negative crossing and $L_{\|}$has no crossing. We set the normalization of $F(L)$ by selecting its value on the unknot to be

$$
F(\text { unknot })=-\frac{t-t^{-1}}{q-q^{-1}}
$$

This normalization corresponds to the multiplicativity condition

$$
F\left(L_{1} \sqcup L_{2}\right)=F\left(L_{1}\right) F\left(L_{2}\right),
$$

where $L_{1} \sqcup L_{2}$ is a disjoint union of two links.

In the original version of this paper the categorification complex satisfied the topological invariance condition (1) and the relation (3) only up to a degree shift. Hao Wu [15] showed that a degree shift related to the first Reidemeister move can be removed if one allows half-integer values for the grading degrees. Here we follow his grading assignment.

\section{Matrix factorizations with a parameter}

Take an oriented arc $c$ as in Figure 1, label its ends $x_{1}$ and $x_{2}$, and assign the potential $a x_{1}-a x_{2}$ to the arc. Let $R=\mathbb{Q}\left[a, x_{1}, x_{2}\right]$ and define $C_{c}$ as the factorization

$$
R \stackrel{a}{\longrightarrow} R \stackrel{x_{1}-x_{2}}{\longrightarrow} R \text {. }
$$


We have $d^{2}=a x_{1}-a x_{2}$ and view $C_{c}$ as an object of the homotopy category of matrix factorizations with the potential $a\left(x_{1}-x_{2}\right)$.

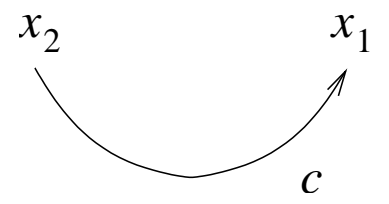

Figure 1: An arc

Make $R$ bigraded by setting

$$
\operatorname{deg}(a)=(2,0), \quad \operatorname{deg}\left(x_{i}\right)=(0,2) .
$$

We refer to these degrees as $t$-degree and $q$-degree respectively. Equation (6) implies $\operatorname{deg}\left(d^{2}\right)=(2,2)$, and we select the bigrading of the middle $R$ in the factorization so that $\operatorname{deg}(d)=(1,1)$ :

$$
R \stackrel{a}{\longrightarrow} R\{-1,1\} \stackrel{x_{1}-x_{2}}{\longrightarrow} R,
$$

where the bidegree shift by $\left(n_{1}, n_{2}\right)$ is denoted $\left\{n_{1}, n_{2}\right\}$.

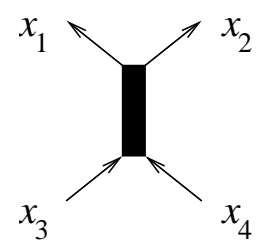

Figure 2: Wide edge $t$

Next, given a wide edge $t$ as in Figure 2, assign variables $x_{1}, x_{2}, x_{3}, x_{4}$ to the edges next to it. We can write

$$
a x_{1}+a x_{2}-a x_{3}-a x_{4}=a\left(x_{1}+x_{2}-x_{3}-x_{4}\right)+0\left(x_{1} x_{2}-x_{3} x_{4}\right) .
$$

Define $C_{t}$ as the tensor product (over $R$ ) of factorizations

$$
R \stackrel{a}{\longrightarrow} R\{-1,1\} \stackrel{x_{1}+x_{2}-x_{3}-x_{4}}{\longrightarrow} R
$$

and

$$
R \stackrel{0}{\longrightarrow} R\{-1,3\} \stackrel{x_{1} x_{2}-x_{3} x_{4}}{\longrightarrow} R
$$


where $R=\mathbb{Q}\left[a, x_{1}, x_{2}, x_{3}, x_{4}\right]$.

Throughout the paper we work with matrix factorizations with potentials $w=a \sum_{i} \epsilon_{i} x_{i}$ where $i$ ranges over some finite set $I$ of integers and $\epsilon_{i} \in\{1,-1\}$ are "orientations" of $x_{i}$. The category $m f_{w}$ has objects $(M, d)$ where $M=M^{0} \oplus M^{1}$ and $M^{0}, M^{1}$ are free bigraded $R$-modules (possibly of infinite rank), while $d$ is a generalized differential

$$
M^{0} \stackrel{d}{\longrightarrow} M^{1} \stackrel{d}{\longrightarrow} M^{0}
$$

of bidegree $(1,1)$ and subject to $d^{2}=w$. Here $R$ is the ring of polynomials in $a$ and $x_{i}$ with rational coefficients. The bidegrees are given by formula (6). Morphisms in $m f_{w}$ are bidegree-preserving maps of $R$-modules $M^{0} \rightarrow N^{0}, M^{1} \rightarrow N^{1}$ that commute with $d$.

We found it useful to visualize a matrix factorization as above by decomposing

$$
M^{0}=\underset{k, l}{\oplus} M_{k, l}^{0}, \quad M^{1}=\underset{k, l}{\oplus} M_{k, l}^{1},
$$

as direct sums of vector spaces, one for each bidegree $(k, l)$, and placing them in the nodes of a coordinate lattice, see Figure 3. Diagonal arrows denote the differential, horizontal arrows show multiplication by $a$ and vertical arrows-multiplications by $x_{i}$.

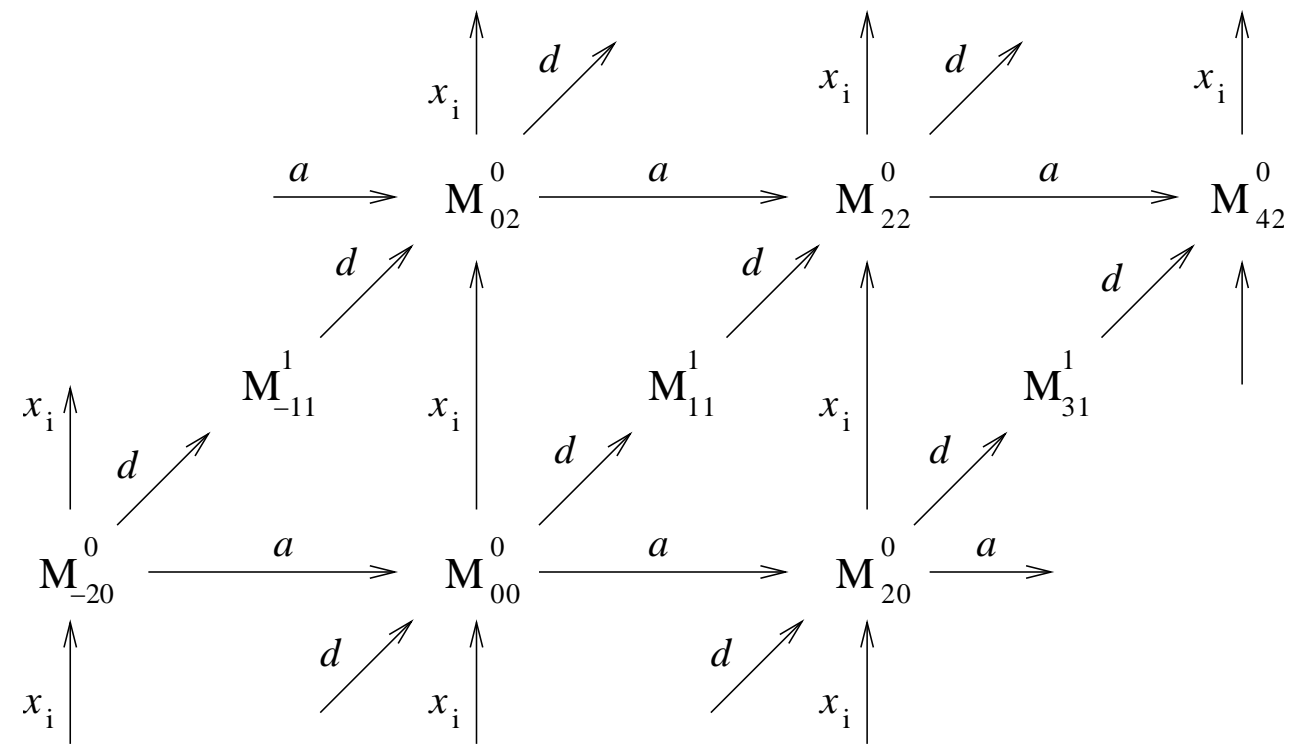

Figure 3: Lattice of factorization $M$ 
The category $h m f_{w}$ of matrix factorizations up to chain homotopies has the same objects as $m f_{w}$ and the $\mathbb{Q}$-vector space of morphisms from $M$ to $N$ is the quotient of the space of morphisms in $m f_{w}$ by null-homotopic morphisms (the homotopy maps must have bidegree $(-1,-1))$.

If the index set $I$ is empty, then $R=\mathbb{Q}[a]$, and $m f_{w}$ is equivalent to the category of complexes of free graded $\mathbb{Q}[a]$-modules.

As the formulas (6), (7), (8) and (9) indicate, in all our matrix factorizations the homological $\mathbb{Z}_{2}$ degree equals $t$-degree modulo 2 . Therefore we will not trace the $\mathbb{Z}_{2}$ degree separately, while keeping in mind that $t$-degree is of homological nature, thus producing a sign factor in the Euler characteristic computation.

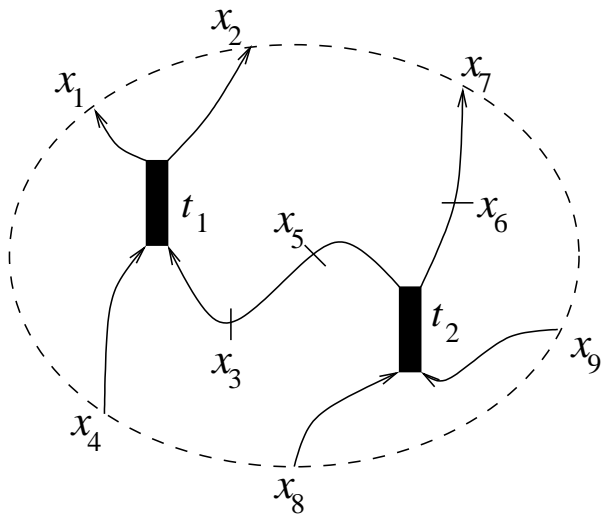

Figure 4: Graph $\Gamma$, an example

In general, given a planar marked graph $\Gamma$ (as described in [8, Introduction]), possibly with boundary points, we assign to it a matrix factorization $C(\Gamma)$ which is the tensor product of $C_{c}$, over all $\operatorname{arcs} c$ in $\Gamma$, and $C_{t}$, over all wide edges $t$ in $\Gamma$. For instance, for the graph in Figure 4,

$$
C(\Gamma)=C_{t_{1}} \otimes C_{t_{2}} \otimes C_{c_{1}} \otimes C_{c_{2}}
$$

where $c_{1}, c_{2}$ are the arcs of $\Gamma$ with endpoints labelled $x_{3}, x_{5}$ and $x_{7}, x_{6}$, respectively. The tensor product is taken over suitable polynomial rings $\mathbb{Q}\left[a, x_{i}\right]$ so that $C(\Gamma)$ is a finite rank free $\mathbb{Q}\left[a, x_{1}, \ldots, x_{9}\right]$-module. The potential

$$
w=a\left(x_{1}+x_{2}-x_{7}-x_{4}-x_{8}-x_{9}\right),
$$

and we view $C(\Gamma)$ as an object of $m f_{w}$ (or $h m f_{w}$ ) with the ground ring $R$ the polynomial ring $\mathbb{Q}\left[a, x_{1}, x_{2}, x_{4}, x_{7}, x_{8}, x_{9}\right]$ in $a$ and external (or boundary) variables. 
The other variables $x_{3}, x_{5}, x_{6}$ are "internal". Notice that $C(\Gamma)$ has infinite rank as an $R$-module.

When $\Gamma$ has no boundary points, $w=0$ and $C(\Gamma)$ becomes a 2-periodic complex

$$
C^{0}(\Gamma) \stackrel{d}{\longrightarrow} C^{1}(\Gamma) \stackrel{d}{\longrightarrow} C^{0}(\Gamma)
$$

of bigraded $\mathbb{Q}[a]$-modules. Its cohomology, denoted $H(\Gamma)$, is a bigraded $\mathbb{Q}[a]$ module.

If $\Gamma$ is a single circle with one mark (glue together the endpoints of the arc in the Figure 1 and place a mark there), the complex is

$$
\mathbb{Q}\left[a, x_{1}\right] \stackrel{a}{\longrightarrow} \mathbb{Q}\left[a, x_{1}\right]\{-1,1\} \stackrel{0}{\longrightarrow} \mathbb{Q}\left[a, x_{1}\right]
$$

(since now $x_{2}=x_{1}$ ), and $H(\Gamma) \cong \mathbb{Q}\left[x_{1}\right]\{-1,1\}$.
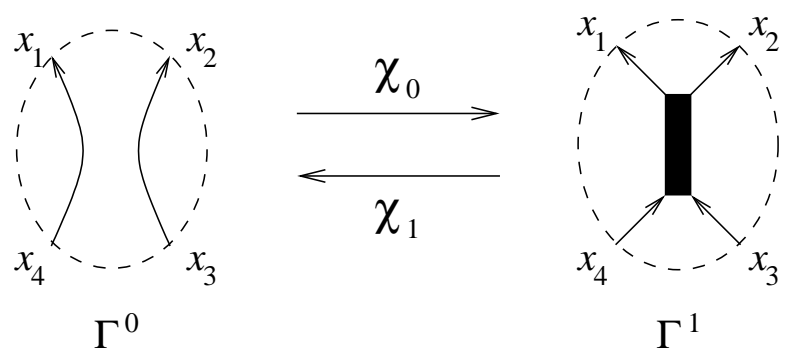

$\Gamma^{1}$

Figure 5: Graphs $\Gamma^{0}$ and $\Gamma^{1}$

Consider the diagrams $\Gamma^{0}, \Gamma^{1}$ in Figure 5. Factorization $C\left(\Gamma^{0}\right)$ is the tensor product of

$$
R \stackrel{a}{\longrightarrow} R\{-1,1\} \stackrel{x_{1}-x_{4}}{\longrightarrow} R
$$

and

$$
R \stackrel{a}{\longrightarrow} R\{-1,1\} \stackrel{x_{2}-x_{3}}{\longrightarrow} R,
$$

where $R=\mathbb{Q}\left[a, x_{1}, x_{2}, x_{3}, x_{4}\right]$. In the product basis, $C\left(\Gamma^{0}\right)$ has the form

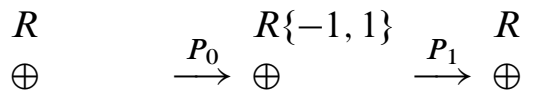

$$
\begin{aligned}
& R\{-2,2\} \quad R\{-1,1\} \quad R\{-2,2\}
\end{aligned}
$$

with

$$
P_{0}=\left(\begin{array}{cc}
a & x_{3}-x_{2} \\
a & x_{1}-x_{4}
\end{array}\right), \quad P_{1}=\left(\begin{array}{cc}
x_{1}-x_{4} & x_{2}-x_{3} \\
-a & a
\end{array}\right)
$$


Likewise, $C\left(\Gamma^{1}\right)$ has the presentation

$$
\begin{array}{lllll}
R & & R\{-1,1\} \\
\oplus & \stackrel{Q_{0}}{\longrightarrow} & \oplus & \stackrel{Q_{1}}{\longrightarrow} & \oplus \\
R\{-2,4\} & R\{-1,3\} & & R\{-2,4\}
\end{array}
$$

with

$$
Q_{0}=\left(\begin{array}{cc}
a & x_{3} x_{4}-x_{1} x_{2} \\
0 & x_{1}+x_{2}-x_{3}-x_{4}
\end{array}\right), \quad Q_{1}=\left(\begin{array}{cc}
x_{1}+x_{2}-x_{3}-x_{4} & x_{1} x_{2}-x_{3} x_{4} \\
0 & a
\end{array}\right) .
$$

A map between $C\left(\Gamma^{0}\right)$ and $C\left(\Gamma^{1}\right)$ can be described by a pair of $2 \times 2$ matrices with coefficients in $R$ that specify the images of the basis vectors of $C^{i}\left(\Gamma^{0}\right)$ in $C^{i}\left(\Gamma^{1}\right)$ for $i=0,1$.

Let $\chi_{0}: C\left(\Gamma^{0}\right) \longrightarrow C\left(\Gamma^{1}\right)$ be given by the pair of matrices

$$
U_{0}^{0}=\left(\begin{array}{cc}
x_{4}-x_{2} & 0 \\
0 & 1
\end{array}\right), \quad U_{0}^{1}=\left(\begin{array}{cc}
x_{4} & -x_{2} \\
-1 & 1
\end{array}\right) .
$$

Our bases in $C\left(\Gamma^{0}\right)$ and $C\left(\Gamma^{1}\right)$ are homogeneous with respect to the bigrading of $R$. It is easy to see that $\chi_{0}$ is a homogeneous map of bidegree $(0,2)$.

Next, define $\chi_{1}: C\left(\Gamma^{1}\right) \longrightarrow C\left(\Gamma^{0}\right)$ by the pair of matrices

$$
U_{1}^{0}=\left(\begin{array}{cc}
1 & 0 \\
0 & x_{4}-x_{2}
\end{array}\right), \quad U_{1}^{1}=\left(\begin{array}{ll}
1 & x_{2} \\
1 & x_{4}
\end{array}\right) .
$$

The map $\chi_{1}$ is bidegree-preserving.

Given a plane diagram $D$ of a tangle, place at least one mark on each internal edge of the diagram (an edge disjoint from the boundary of $D$ ), and label the marks and boundary points by $x_{1}, \ldots, x_{m}$. To each crossing $p$ of the diagram assign the complex $C_{p}$ of matrix factorizations as follows. Up to shifts, the complex is the cone of the map $\chi_{0}$ or $\chi_{1}$, depending on whether the crossing is positive or negative. The shifts are shown in Figure 6.

Thus, if the crossing is positive,

$$
C_{p}=0 \longrightarrow C\left(\Gamma^{0}\right)_{-\frac{1}{2}}\left\{-\frac{1}{2}, \frac{3}{2}\right\} \stackrel{\chi_{0}}{\longrightarrow} C\left(\Gamma^{1}\right)_{\frac{1}{2}}\left\{-\frac{1}{2},-\frac{1}{2}\right\} \longrightarrow 0,
$$

with $C\left(\Gamma^{0}\right)$ positioned in cohomological degree $-\frac{1}{2}$ and $C\left(\Gamma^{1}\right)$ positioned in cohomological degree $\frac{1}{2}$. If the crossing is negative,

$$
C_{p}=0 \longrightarrow C\left(\Gamma^{1}\right)_{-\frac{1}{2}}\left\{\frac{1}{2},-\frac{3}{2}\right\} \stackrel{\chi_{1}}{\longrightarrow} C\left(\Gamma^{0}\right)_{\frac{1}{2}}\left\{\frac{1}{2},-\frac{3}{2}\right\} \longrightarrow 0 .
$$




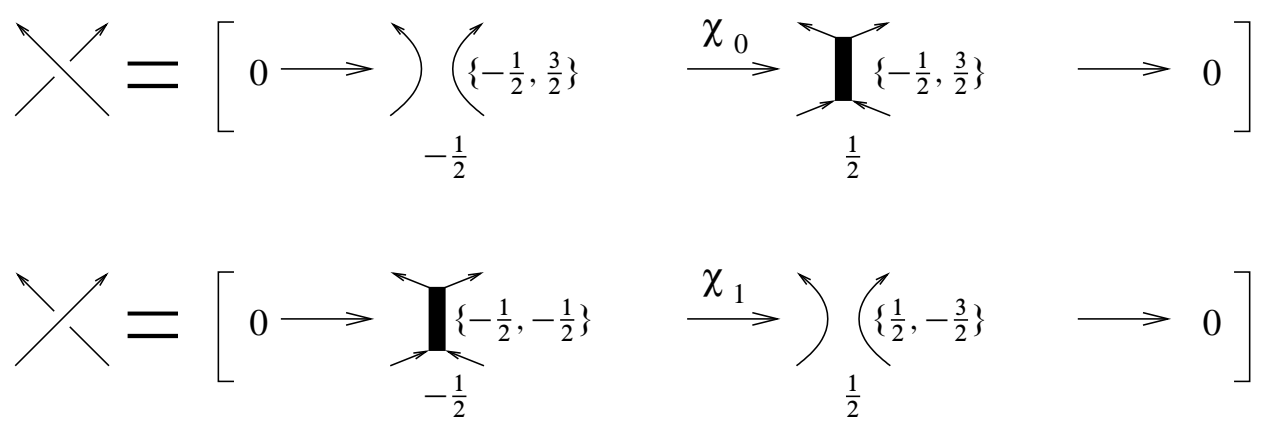

Figure 6: Complex assigned to a crossing

Bidegree shifts make the differential preserve the bidegree and are also required for the Reidemeister move invariance. The half-integer values for the cohomological degrees do not pose a sign problem, because the $t$-degree is also cohomological and half-integer so that the combined cohomological degree stays integer.

Define $C(D)$ as the tensor product of $C_{p}$, over all crossings $p$ of $D$, and $C_{c}$, over all $\operatorname{arcs} c$. It is a complex built out of matrix factorizations $C(\Gamma)$, over all resolutions $\Gamma$ of $D$. The differential $\partial$ preserves the bigrading of each term $C^{j}(D)$. We view $C(D)$ as an object of the category $K\left(h m f_{w}\right)$. The latter is the category whose objects are complexes of objects in $h m f_{w}$ and whose morphisms are homomorphism of complexes modulo null-homotopic morphisms.

Now we specialize to the case when $D$ is a link diagram (has empty boundary). Then each term $C^{j}(D)$ in the complex $C(D)$ is an object of the homotopy category of bigraded free $\mathbb{Q}[a]$-modules. $C^{j}(D)$, for any diagram $D$, decomposes as a direct sum of contractible pieces

$$
0 \longrightarrow \mathbb{Q} \stackrel{1}{\longrightarrow} \mathbb{Q} \longrightarrow 0
$$

and the cohomology $H\left(C^{j}(D)\right)$, which we denote $C H^{j}(D)$. Moreover, $a$ acts trivially on $C H^{j}(D)$, so we can ignore the $\mathbb{Q}[a]$-module structure and think of it as a bigraded $\mathbb{Q}$-vector space,

$$
C H^{j}(D)=\underset{k, l}{\oplus} C H_{k, l}^{j}(D) .
$$

The bigrading descends from the bigrading on matrix factorizations $C(\Gamma)$.

Thus, to $D$ we assign the complex $C H(D)$ of bigraded $\mathbb{Q}$-vector spaces

$$
\cdots \stackrel{\partial}{\longrightarrow} C H^{j}(D) \stackrel{\partial}{\longrightarrow} C H^{j+1}(D) \stackrel{\partial}{\longrightarrow} \cdots
$$


As a $\mathbb{Q}$-vector space, $C H(D)$ is the direct sum of cohomology groups $H(\Gamma)$ of complexes $C(\Gamma)$, over all resolutions of $D$.

For the reasons that we will explain shortly, we consider only link diagrams which are braid closures (see Figure 7).

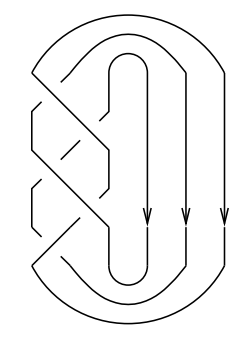

Figure 7: A braid diagram

With a slight abuse of notation we use the same symbol $D$ to denote the braid and the diagram of its closure.

Let $D$ be an $n$-strand braid closure diagram. We associate to it a grading-corrected complex

$$
\widetilde{C H}(D)=C H(D)\left\{\frac{n}{2}, \frac{n}{2}\right\}\left[\frac{n}{2}\right] .
$$

(see Wu's grading assignment [15]). The cohomology $H(D)=H(\widetilde{C H}(D), \partial)$ of the above complex is triply-graded,

$$
H(D)=\underset{j, k, l}{\oplus} H_{k, l}^{j}(D) .
$$

Of course, for the whole construction to be interesting, $H(D)$ should not depend on the choice of a marked diagram $D$, which represents a given oriented link $L$. More precisely, if two diagrams $D_{1}$ and $D_{2}$ represent the same link $L$, then the corresponding categorification complexes $\widetilde{C H}\left(D_{1}\right)$ and $\widetilde{C H}\left(D_{2}\right)$ should be homotopy equivalent, that is, isomorphic as objects of the category $K\left(h m f_{w}\right)$. We begin with the independence of marking placements.

Proposition 1 Let $D$ be a marked tangle diagram. Then $C(D)$, as an object of $K\left(h m f_{w}\right)$, does not depend on the number of markings on each edge of $D$.

Proposition 1 is proved in the next section. 
Type I

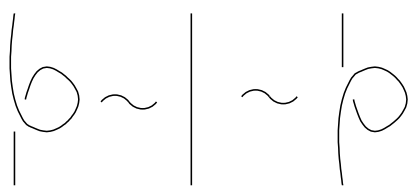

Type IIa

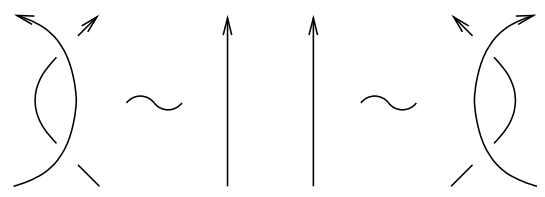

Type IIb

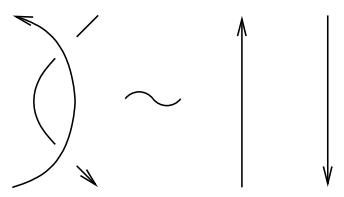

Figure 8: Reidemeister moves

Now consider unmarked diagrams. Two diagrams represent the same link if they can be transformed into each other by a sequence of Reidemeister moves depicted in Figure 8.

Since each Reidemeister move is local, it is sufficient to prove that for each pair of Reidemeister tangles $D_{1}$ and $D_{2}$ their complexes $C\left(D_{1}\right)$ and $C\left(D_{2}\right)$ are homotopy equivalent. Here we encounter an obstacle: we can not prove the invariance under the Reidemeister move IIb. In order to circumvent this problem, we limit ourselves to braid closure diagrams. Then it is sufficient to establish the homotopy equivalence of Reidemeister pairs only for the moves I, IIa and III, all strands in move III being oriented in the same direction. Indeed, two braid diagrams represent the same braid if they can be transformed into each other by braid group relations

$$
\begin{gathered}
\sigma_{i} \sigma_{j}=\sigma_{j} \sigma_{i}, \quad \text { if }|i-j|>1, \\
\sigma_{i} \sigma_{i}^{-1}=I, \quad \sigma_{i}^{-1} \sigma_{i}=I, \\
\sigma_{i} \sigma_{i+1} \sigma_{i}=\sigma_{i+1} \sigma_{i} \sigma_{i+1} .
\end{gathered}
$$

Relation (15) for complexes is satisfied automatically, because both sides correspond to the same diagram. Relations (16) correspond to Reidemeister moves IIa and relation (17) correspond to the Reidemeister move III. The closures of two braids produce the same 
oriented link if the braids are related by two types of Markov moves

$$
\begin{gathered}
D D^{\prime} \leftrightarrow D^{\prime} D \quad \text { (conjugation), } \\
D \leftrightarrow D \sigma_{n}^{ \pm 1}, \quad \text { where } D \text { is an } n \text {-strand braid. }
\end{gathered}
$$

Relation (18) is satisfied automatically, because both closure diagrams are the same, while relation (19) corresponds to the Reidemeister move I.

Proposition 2 If a pair of diagrams $D_{1}$ and $D_{2}$ is a Reidemeister pair of type I, IIa or III then the complexes $C\left(D_{1}\right)$ and $C\left(D_{2}\right)$ are isomorphic as objects of the category $K\left(h m f_{w}\right)$, except in the case of move I, where there is a degree shift:

$$
C\left(D_{1}\right) \simeq C\left(D_{2}\right)\left\{\frac{1}{2}, \frac{1}{2}\right\}\left[\frac{1}{2}\right],
$$

$D_{1}$ being a straight line and $D_{2}$ being a line with a loop.

For a proof see Section 3.

Proposition 2 implies the following theorem.

Theorem 1 Given two braid diagrams $D_{1}, D_{2}$ of an oriented link $L$, the cohomology groups $H\left(D_{1}\right)$ and $H\left(D_{2}\right)$ are isomorphic as triply-graded vector spaces.

Note that the degree shift in formula (20) matches the braid closure degree shift (14), since the diagram $D_{2}$ involves one strand closure.

The graded Euler characteristic of the complex $\widetilde{C H}(D)$ is defined by the formula (2) as a power series in $q$ with coefficients in $\mathbb{Z}\left[t, t^{-1}\right]$. Since the differential $\partial$ of the categorification complex has zero bidegree, one can replace the dimensions of the graded chain vector spaces with the Betti numbers:

$$
\langle D\rangle=\sum_{j, k, l}(-1)^{j+k} t^{2 k} q^{k+l} \operatorname{dim}_{\mathbb{Q}} H_{k, l}^{j}(D) .
$$

At the end of Section 3 we will prove the following theorem.

Theorem 2 For any braid diagram $D$ the Euler characteristic $\langle D\rangle$ of $H(D)$ is related to the HOMFLYPT polynomial of its closure according to formula (3).

Specializing to $t=1$ in the equation (4) nets us the Alexander polynomial. Homologically, $t, q$ and the minus sign correspond to the three grading directions. Hence, suitably collapsing the tri-grading to a bigrading we get a categorification of the Alexander polynomial. 
S Gukov, A Schwarz and C Vafa recently conjectured [6] that there exist integer-valued link invariants $D_{Q, s, r}$ depending on three integer parameters $Q, s, r$, that can be used to determine ranks of sl(n) link homology groups as well as the coefficients of the HOMFLYPT polynomial of a link. These invariants should come from the physical theory of the BPS states and should be related to ranks of cohomology groups of suitable moduli spaces. It would be interesting to try relating $D_{Q, s, r}$ to the dimensions of cohomology groups $H_{k, l}^{j}$. Our normalization of the HOMFLYPT polynomial is similar to the one in [6], both having $q-q^{-1}$ as the denominator of the unknot invariant.

On the other hand, it was independently suggested by several people, including O Viro [14], that there should exist a triply-graded link homology theory with the HOMFLYPT polynomial as the Euler characteristic. The current paper resulted from our search for such a theory and for a combinatorial categorification of the Alexander polynomial.

Triply-graded cohomology theories had previously appeared in the work of Asaeda, Przytycki and Sikora [1] on categorification of invariants of links in I-bundles over surfaces, and in Audoux and Fiedler [2], who introduced a refined Jones polynomial and its categorification, which are only invariant under braid-like isotopies. Restriction to braid-like isotopies appears in our construction as well, but we do not know how our invariant relates to those of [1] and [2].

To conclude this section, we mention several modifications, potential generalizations and illnesses of the homology theory $H$.

- It is not natural that we have to restrict to braid diagrams to get a link invariant. In another sign of disfunctionality, the theory does not extend to all cobordisms. For instance, the cohomology groups of the unknot do not have a Frobenius algebra structure over the cohomology ring of the empty link (it's convenient to define the latter ring to be $\mathbb{Q}[a]$ ), preventing us from extending the theory even to unknotted cobordisms between unlinks.

- Any field $k$ can be used instead of $\mathbb{Q}$. More generally, we can work over $\mathbb{Z}$, so that the invariant of a closed planar graph $\Gamma$ is a complex of graded free abelian groups, up to chain homotopy equivalence, and the invariant of a link is a complex of complexes as above, up to chain homotopy equivalence. Taking the homology $H(\Gamma, \mathbb{Z})$ of each resolution of $D$ and forming a complex out of them produces a complex $C H(D, \mathbb{Z})$ from a diagram $D$. We then specialize to braid diagrams and take the cohomology of $C H(D, \mathbb{Z})$. The resulting groups $H(D, \mathbb{Z})$ are triply-graded and, up to isomorphism, do not depend on the choice of braid diagram $D$, given $L$.

- In Section 3 we rewrite the factorizations $C\left(\Gamma^{0}\right), C\left(\Gamma^{1}\right)$ and the maps $\chi_{0}, \chi_{1}$ in the form that depends only on $a$ and the differences $x_{i}-x_{j}$ of the variables $x_{i}$. 
This allows us to pass from the ring $R=\mathbb{Q}\left[a, x_{1}, \ldots, x_{m}\right]$ to the smaller ring $\bar{R}=\mathbb{Q}\left[a, x_{2}-x_{1}, \ldots, x_{m}-x_{1}\right]$. The definition of cohomology and the proof of its invariance work over $\bar{R}$ as well, leading to reduced cohomology groups $\bar{H}(D)$, with the property $H(D)=\bar{H}(D) \otimes \mathbb{Q} \mathbb{Q}[x]$. In the reduced theory the unknot has one-dimensional cohomology groups.

- $s l(n)$ link homology theory (see [8]) utilized the potential $x^{n+1}$. Soon afterwards Gornik [5] studied a deformation of that theory with the potential $x^{n+1}-(n+$ 1) $\beta^{n} x$. In the $n=2$ case the deformation was found earlier by Lee [9] and used by Rasmussen in his combinatorial proof of the Milnor conjecture [13]. The definitions in [8] can be generalized to the potential $x^{n+1}+a_{n} x^{n}+\cdots+a_{1} x$ where $a_{1}, \ldots, a_{n}$ are formal variables. We hope that this generalization will be invariant under the Reidemeister moves and will turn out to be the " $s l(n)-$ equivariant" version of $s l(n)$ link homology. The invariant of the empty link should be the ring of polynomials in $a_{1}, \ldots, a_{n}$, and naturally isomorphic to the $U(n)$-equivariant cohomology ring of the point. The invariant of the unknot should be the quotient of the polynomial ring $\mathbb{Q}\left[x, a_{1}, \ldots, a_{n}\right]$ by the relation $x^{n+1}+a_{n} x^{n}+\cdots+a_{1} x=0$, isomorphic to the $U(n)$-equivariant cohomology ring of $\mathbb{C} \mathbb{P}^{n}$. A certain version of Bar-Natan link homology [3] from Khovanov [7] should correspond to the potential $x^{3}+a x$.

- For a common generalization of the $U(n)$-equivariant link homology and the theory described here one could try the potential $a_{n+1} x^{n+1}+a_{n} x^{n}+\cdots+a_{1} x$ with all $a_{i}$ being formal variables. Factorizations $C\left(\Gamma^{0}\right), C\left(\Gamma^{1}\right)$, the maps $\chi_{0}, \chi_{1}$ and the complex $C(D)$ can be defined for this potential as well, but we do not know whether this theory will be invariant under the Reidemeister-Markov moves of braid diagrams.

\section{Proofs}

\subsection{Product factorizations, graph homology and Koszul complexes}

Given a polynomial ring $R$ and a pair of elements $a_{1}, b_{1} \in R$, we denote by $\left(a_{1}, b_{1}\right)$ the factorization

$$
R \stackrel{a_{1}}{\longrightarrow} R \stackrel{b_{1}}{\longrightarrow} R \text {. }
$$

Given a finite set of such pairs $\left(a_{i}, b_{i}\right), 1 \leq i \leq n$, we denote by $(\mathbf{a}, \mathbf{b})$ their tensor product (over $R$ ):

$$
(\mathbf{a}, \mathbf{b}) \stackrel{\text { def }}{=} \otimes_{i}\left(a_{i}, b_{i}\right), \quad \mathbf{a}=\left(a_{1}, \ldots, a_{n}\right), \quad \mathbf{b}=\left(b_{1}, \ldots, b_{n}\right) .
$$


We will also write $(\mathbf{a}, \mathbf{b})$ in the column form

$$
\left(\begin{array}{cc}
a_{1} & b_{1} \\
a_{2} & b_{2} \\
\vdots & \vdots \\
a_{n} & b_{n}
\end{array}\right)
$$

and call it a Koszul factorization. By an elementary transformation of rows $i$ and $j$ we mean a modification

$$
\left(\begin{array}{ll}
a_{i} & b_{i} \\
a_{j} & b_{j}
\end{array}\right) \stackrel{[i j]_{\lambda}}{\longrightarrow}\left(\begin{array}{cc}
a_{i} & b_{i}+\lambda b_{j} \\
a_{j}-\lambda a_{i} & b_{j}
\end{array}\right)
$$

for some $\lambda \in R$. We denote it by $[i j]_{\lambda}$. All other rows of $(\mathbf{a}, \mathbf{b})$ are left unchanged. An elementary transformation takes a Koszul factorization $(\mathbf{a}, \mathbf{b})$ to an isomorphic factorization, since we are only changing a basis vector in the free $R$-module underlying the factorization $(\mathbf{a}, \mathbf{b})$.

Suppose now that $y$ is one of the generators of the polynomial ring $R$, so we can write $R=R^{\prime}[y]$, and that the potential $w=\sum a_{i} b_{i}$ lies in $R^{\prime}$ (in this situation we say that $y$ is an internal variable). Then any factorization $M$ over $R$ restricts to (an infinite rank) factorization over $R^{\prime}$, which we denote $M^{\prime}$. Assume furthermore that one of the rows in $(\mathbf{a}, \mathbf{b})$ has the form $(0, y-\mu)$ where $\mu \in R^{\prime}$. Denote by $\left(\mathbf{a}^{\prime}, \mathbf{b}^{\prime}\right)$ the factorization over $R^{\prime}$ obtained from $(\mathbf{a}, \mathbf{b})$ by removing the row $(0, y-\mu)$ and substituting $\mu$ for $y$ everywhere in all other rows.

Proposition 3 Factorizations $\left(\mathbf{a}^{\prime}, \mathbf{b}^{\prime}\right)$ and $(\mathbf{a}, \mathbf{b})^{\prime}$ are chain homotopy equivalent.

Proof By changing a variable $y \rightarrow y-\mu$ we reduce to the case $\mu=0$. We can write $a_{i}=a_{i}^{\prime}+y a_{i}^{\prime \prime}$ and $b_{i}=b_{i}^{\prime}+y b_{i}^{\prime \prime}$ where $a_{i}^{\prime}, b_{i}^{\prime} \in R^{\prime}$. Applying elementary transformations to rows $(0, y)$ and $\left(a_{i}, b_{i}\right)$ we reduce the latter to $\left(a_{i}, b_{i}^{\prime}\right)$, while $(0, y)$ is transformed into $\left(\sum a_{i} b_{i}^{\prime \prime}, y\right)$. Next, change $\left(\sum a_{i} b_{i}^{\prime \prime}, y\right)$ into $\left(y, \sum a_{i} b_{i}^{\prime \prime}\right)$ (this shifts factorization $M$ to $M\langle 1\rangle$ ) and apply elementary transformations to rows $\left(a_{i}, b_{i}^{\prime}\right)$ and $\left(y, \sum a_{i} b_{i}^{\prime \prime}\right)$. The row $\left(a_{i}, b_{i}^{\prime}\right)$ becomes $\left(a_{i}^{\prime}, b_{i}^{\prime}\right)$, while the row with $y$ turns into $\left(y, \sum\left(a_{i} b_{i}^{\prime \prime}+a_{i}^{\prime \prime} b_{i}^{\prime}\right)\right)$. Since the potential does not depend on $y$, the latter sum is zero. Now shift $(y, 0)$ back to $(0, y)$. The result is the Koszul factorization, isomorphic to $(\mathbf{a}, \mathbf{b})$, with rows $\left(a_{i}^{\prime}, b_{i}^{\prime}\right)$ and $(0, y)$. This factorization is the tensor product of $\left(\mathbf{a}^{\prime}, \mathbf{b}^{\prime}\right)$, as defined above, and $(0, y)$. Therefore, $(\mathbf{a}, \mathbf{b})$ is isomorphic, as an $R$-factorization, to the total factorization of the bifactorization

$$
\left(\mathbf{a}^{\prime}, \mathbf{b}^{\prime}\right) \otimes_{R^{\prime}} R^{\prime}[y] \stackrel{0}{\longrightarrow}\left(\mathbf{a}^{\prime}, \mathbf{b}^{\prime}\right) \otimes_{R^{\prime}} R^{\prime}[y] \stackrel{y}{\longrightarrow}\left(\mathbf{a}^{\prime}, \mathbf{b}^{\prime}\right) \otimes_{R^{\prime}} R^{\prime}[y] .
$$


As a factorization over the smaller ring $R^{\prime}$, it decomposes into a direct sum of contractible factorizations which are the total factorizations of

$$
\left(\mathbf{a}^{\prime}, \mathbf{b}^{\prime}\right) \otimes y^{j+1} \stackrel{0}{\longrightarrow}\left(\mathbf{a}^{\prime}, \mathbf{b}^{\prime}\right) \otimes y^{j} \stackrel{y}{\longrightarrow}\left(\mathbf{a}^{\prime}, \mathbf{b}^{\prime}\right) \otimes y^{j+1},
$$

for $j \geq 0$, and the factorization $\left(\mathbf{a}^{\prime}, \mathbf{b}^{\prime}\right)$. The proposition follows.

Remark The second half of the above proof just says that the complex of $R^{\prime}$-modules

$$
0 \longrightarrow R^{\prime}[y] \stackrel{y}{\longrightarrow} R^{\prime}[y] \longrightarrow 0
$$

is the direct sum of contractible complexes

$$
0 \longrightarrow R^{\prime} y^{j} \stackrel{y}{\longrightarrow} R^{\prime} y^{j+1} \longrightarrow 0
$$

and the complex $0 \longrightarrow R^{\prime} \longrightarrow 0$.

Suppose we are given a planar marked graph $\Gamma$, possibly with boundary. To $\Gamma$ we assigned a Koszul factorization $C(\Gamma)$ which has a rather special form. Each arc in $\Gamma$ contributes the row $\left(a, x_{i}-x_{j}\right)$ to the Koszul matrix of $C(\Gamma)$, where $x_{i}$ and $x_{j}$ are the labels at the endpoints of the arc. Each wide edge in $\Gamma$ contributes two rows

$$
\left(\begin{array}{cc}
a & x_{i}+x_{j}-x_{k}-x_{l} \\
0 & x_{i} x_{j}-x_{k} x_{l}
\end{array}\right)
$$

to the Koszul matrix, where $x_{i}, x_{j}, x_{k}, x_{l}$ are the labels bounding the edge. If $\Gamma$ has $m_{1}$ arcs and $m_{2}$ wide edges, the Koszul matrix of $C(\Gamma)$ will have $n=m_{1}+2 m_{2}$ rows. Permute these rows so that the first $m_{1}+m_{2}$ rows have the form $(a, z)$ where the $z$ are some linear functions of the $x_{i}$. We call these rows linear rows. The last $m_{2}$ rows have the form $\left(0, x_{i} x_{j}-x_{k} x_{l}\right)$ for various quadruples of indices $(i, j, k, l)$. Call these quadratic rows.

Apply elementary transformations with $\lambda=1$ to the first row paired with every other linear row. In other words, we convert $b_{1}$ to $b_{1}+b_{2}+\cdots+b_{m_{1}+m_{2}}$ and subtract $a_{1}=a$ from $a_{p}=a$ for $p=2,3, \ldots, m_{1}+m_{2}$. The Koszul matrix transforms into a matrix with the first row $\left(a, \sum \epsilon_{i} x_{i}\right)$ where the sum is over all boundary points of $\Gamma$ and $\epsilon_{i}= \pm 1$ depending on the orientation of $\Gamma$ at that point. All other linear rows acquire the form $(0, z)$, with the same linear functions $z$ as before. Nothing happens to the quadratic rows. The Koszul matrix now has the form

$$
\left(\begin{array}{cc}
a & b_{1} \\
0 & b_{2} \\
\vdots & \vdots \\
0 & b_{n}
\end{array}\right)
$$


with $b_{1}=\sum \epsilon_{i} x_{i}$. After this change of basis, every row but the first one has the first term zero. Hence, it comes from a one-term Koszul complex

$$
0 \longrightarrow R \stackrel{b_{p}}{\longrightarrow} R \longrightarrow 0
$$

by collapsing cohomological grading from $\mathbb{Z}$ to $\mathbb{Z}_{2}$. Likewise, the tensor product of all rows save the first is a factorization obtained from the Koszul complex of the sequence $\left(b_{2}, b_{3}, \ldots, b_{n}\right)$ by collapsing the grading.

Note that our polynomial ring is, in addition, bigraded. Taking all gradings into account, the collapse is from a triple grading to a bigrading (see Figure 3 ). No cyclic components appear in the collapsed grading since the differential has nonzero bidegree $(1,1)$. Finally, observe that in the new Koszul matrix parameter $a$ appears only once, in the first row.

Next consider the case when $\Gamma$ is closed (has no boundary points). The first row becomes $\left(\begin{array}{ll}a & 0\end{array}\right)$ and the whole factorization comes from the Koszul complex of the sequence $\left(a, b_{2}, \ldots, b_{n}\right)$ by collapsing its grading. Moreover, $a$ plays a purely decorative role, and, using Proposition 3, we can throw out this row simultaneously with removing $a$ from the list of variables, which would then have only the $x_{i}$. In other words, the cohomology $H(\Gamma)$ of the factorization $C(\Gamma)$ is isomorphic to the cohomology of the Koszul complex of the sequence $\left(b_{2}, b_{3}, \ldots, b_{n}\right)$, with the trigrading collapsed to a bigrading.

Thus, although the 2-periodic complex $C(\Gamma)$ as well as its cohomology $H(\Gamma)$ are $\mathbb{Q}[a]$-modules, $a$ acts trivially on $H(\Gamma)$.

\subsection{Maps $\chi_{0}, \chi_{1}$ revisited}

Recall the row operation $[i j]_{\lambda}$ on a Koszul matrix of a factorization:

$$
\left(\begin{array}{ll}
a_{i} & b_{i} \\
a_{j} & b_{j}
\end{array}\right) \longrightarrow\left(\begin{array}{cc}
a_{i} & b_{i}+\lambda b_{j} \\
a_{j}-\lambda a_{i} & b_{j}
\end{array}\right)
$$

Denote by $|0\rangle$ and $|1\rangle$ the standard basis vectors in factorizations $\left(a_{i}, b_{i}\right)$ and $\left(a_{j}, b_{j}\right)$ :

$$
\begin{aligned}
& R|0\rangle \stackrel{a_{i}}{\longrightarrow} R|1\rangle \stackrel{b_{i}}{\longrightarrow} R|0\rangle, \\
& R|0\rangle \stackrel{a_{j}}{\longrightarrow} R|1\rangle \stackrel{b_{j}}{\longrightarrow} R|0\rangle .
\end{aligned}
$$

Let $|00\rangle,|01\rangle,|10\rangle,|11\rangle$ be the standard basis vectors in the tensor product factorization $\left(a_{i}, b_{i}\right) \otimes\left(a_{j}, b_{j}\right)$. The row operation $[i j]_{\lambda}$ corresponds to the isomorphism of factorizations

$$
\left(a_{i}, b_{i}\right) \otimes\left(a_{j}, b_{j}\right) \cong\left(a_{i}, b_{i}+\lambda b_{j}\right) \otimes\left(a_{j}-\lambda a_{i}, b_{j}\right)
$$


which takes the standard basis of the LHS factorization to the basis

$$
|00\rangle,|01\rangle,|10\rangle-\lambda|01\rangle,|11\rangle
$$

of the RHS tensor product.

Denote by $\psi(y)$ the following morphism between two Koszul factorizations:

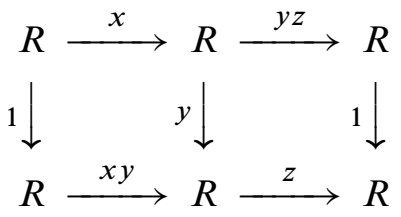

Lemma 1 The following squares are commutative:

$$
\begin{aligned}
& \left(\begin{array}{cc}
a_{1} & b_{1} \\
a_{2} & b_{2} c_{2}
\end{array}\right) \quad \stackrel{\operatorname{Id} \otimes \psi\left(c_{2}\right)}{\longrightarrow} \quad\left(\begin{array}{cc}
a_{1} & b_{1} \\
a_{2} c_{2} & b_{2}
\end{array}\right) \\
& {[12]_{\lambda} \downarrow \quad[12]_{\lambda c_{2} \downarrow} \downarrow} \\
& \left(\begin{array}{cc}
a_{1} & b_{1}+\lambda b_{2} c_{2} \\
a_{2}-\lambda a_{1} & b_{2} c_{2}
\end{array}\right) \stackrel{\operatorname{Id} \otimes \psi\left(c_{2}\right)}{\longrightarrow}\left(\begin{array}{cc}
a_{1} & b_{1}+\lambda b_{2} c_{2} \\
\left(a_{2}-\lambda a_{1}\right) c_{2} & b_{2}
\end{array}\right) \\
& \left(\begin{array}{cc}
a_{1} & b_{1} \\
a_{2} & b_{2} c_{2}
\end{array}\right) \quad \stackrel{\operatorname{Id} \otimes \psi\left(c_{2}\right)}{\longrightarrow} \quad\left(\begin{array}{cc}
a_{1} & b_{1} \\
a_{2} c_{2} & b_{2}
\end{array}\right) \\
& {[21]_{\lambda c_{2} \downarrow} \downarrow[21]_{\lambda} \downarrow} \\
& \left(\begin{array}{cc}
a_{1}-\lambda c_{2} a_{2} & b_{1} \\
a_{2} & c_{2}\left(b_{2}+\lambda b_{1}\right)
\end{array}\right) \stackrel{\operatorname{Id} \otimes \psi\left(c_{2}\right)}{\longrightarrow}\left(\begin{array}{cc}
a_{1}-\lambda c_{2} a_{2} & b_{1} \\
a_{2} c_{2} & b_{2}+\lambda b_{1}
\end{array}\right)
\end{aligned}
$$

Proof The proof follows by direct computation.

Denote by $\psi^{\prime}(y)$ the "opposite" morphism of $\psi(y)$ :

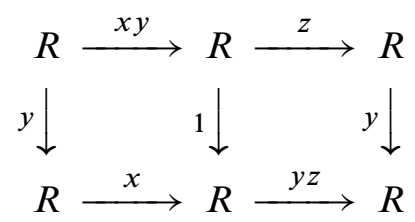

The analogue of Lemma 1 holds for $\psi^{\prime}$ as well (just reverse all horizontal arrows in the commutative diagrams above). We call $\psi$ and $\psi^{\prime}$ flip morphisms.

Starting with the Koszul matrices for $C\left(\Gamma^{0}\right)$ and $C\left(\Gamma^{1}\right)$ and applying a row transformation to each of them, we get the following equivalent Koszul forms for these 
factorizations:

$$
\begin{aligned}
& C\left(\Gamma^{0}\right): \quad\left(\begin{array}{cc}
a & x_{1}-x_{4} \\
a & x_{2}-x_{3}
\end{array}\right) \stackrel{[12]_{1}}{\longrightarrow}\left(\begin{array}{cc}
a & x_{1}+x_{2}-x_{3}-x_{4} \\
0 & x_{2}-x_{3}
\end{array}\right) \\
& C\left(\Gamma^{1}\right):\left(\begin{array}{cc}
a & x_{1}+x_{2}-x_{3}-x_{4} \\
0 & x_{1} x_{2}-x_{3} x_{4}
\end{array}\right) \stackrel{[21]_{-x_{2}}}{\longrightarrow}\left(\begin{array}{cc}
a & x_{1}+x_{2}-x_{3}-x_{4} \\
0 & \left(x_{2}-x_{3}\right)\left(x_{4}-x_{2}\right)
\end{array}\right)
\end{aligned}
$$

The first rows of these new Koszul matrices for $C\left(\Gamma^{0}\right), C\left(\Gamma^{1}\right)$ are identical while the second rows look related. In fact, there is a flip morphism $\psi\left(x_{4}-x_{2}\right)$ from $\left(0,\left(x_{2}-x_{3}\right)\left(x_{4}-x_{2}\right)\right)$ to $\left(0, x_{2}-x_{3}\right)$ :

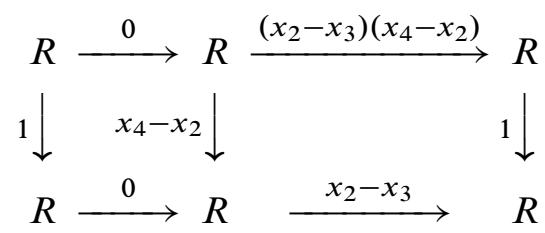

and the flip morphism $\psi^{\prime}\left(x_{4}-x_{2}\right)$ back. Tensoring these flip morphisms with the identity morphism on the first row, we obtain maps of factorizations

$$
\mathrm{Id} \otimes \psi^{\prime}\left(x_{4}-x_{2}\right): C\left(\Gamma^{0}\right) \longrightarrow C\left(\Gamma^{1}\right), \quad \operatorname{Id} \otimes \psi\left(x_{4}-x_{2}\right): C\left(\Gamma^{1}\right) \longrightarrow C\left(\Gamma^{0}\right) .
$$

Lemma 2 Maps Id $\otimes \psi^{\prime}\left(x_{4}-x_{2}\right)$ and Id $\otimes \psi\left(x_{4}-x_{2}\right)$ are equal to $\chi_{0}$ and $\chi_{1}$, respectively.

Proof The proof is a straightforward linear algebra computation.

Therefore, our definition of the complex $C(D)$ of factorizations assigned to a tangle diagram can be rewritten via modified Koszul matrices as above and maps $\psi, \psi^{\prime}$. We will use this alternative presentation in our proof of the invariance of $C(D)$ below. The new definition simplifies the appearance of $C(D)$ by creating more zeros in the Koszul matrices of $C(\Gamma)$ and making the differential easier to describe and understand (at the cost of breaking the "lateral" symmetry $x_{1} \leftrightarrow x_{2}, x_{3} \leftrightarrow x_{4}$ of the original Koszul matrices). The differential acts now as the identity on all but $m_{2}$ rows, where $m_{2}$ is the number of crossings of $D$.

\subsection{Markings do not matter}

To define the complex $C(D)$ for a tangle diagram $D$, we need to place several marks on $D$ : at least one on each internal edge and each circle and some (possibly none) on each external edge (an edge containing a boundary point). In this subsection we prove Proposition 1 that was stated earlier and says that, up to chain homotopy equivalence, $C(D)$ does not depend on how marks are placed on the edges of $D$. 

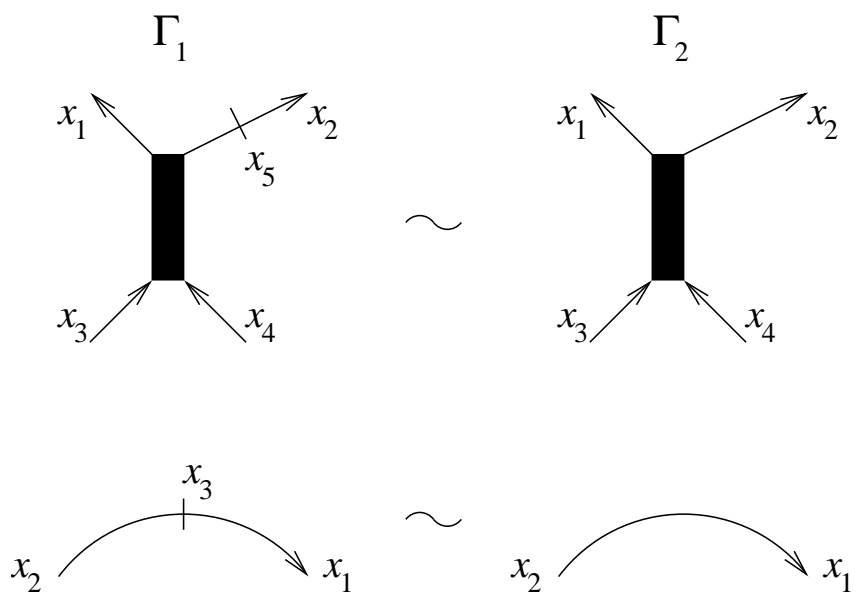

Figure 9: Mark removal equivalences

Lemma 3 Factorizations $\Gamma_{1}$ and $\Gamma_{2}$ are isomorphic in $h m f_{w}$ if $\Gamma_{2}$ is obtained from $\Gamma_{1}$ by removing a mark.

Proof It is enough to check this property locally. We depicted two such local pairs $\left(\Gamma_{1}, \Gamma_{2}\right)$ in Figure 9, and refer the reader to [8] for a more detailed treatment. We only check the isomorphism for the top pair in Figure 9, other cases are similar. We transform the Koszul matrix of $C\left(\Gamma_{1}\right)$ as follows:

$$
\left(\begin{array}{cc}
a & x_{1}+x_{5}-x_{3}-x_{4} \\
0 & x_{1} x_{5}-x_{3} x_{4} \\
a & x_{2}-x_{5}
\end{array}\right) \stackrel{[13]_{1}}{\longrightarrow}\left(\begin{array}{cc}
a & x_{1}+x_{2}-x_{3}-x_{4} \\
0 & x_{1} x_{5}-x_{3} x_{4} \\
0 & x_{2}-x_{5}
\end{array}\right)
$$

The variable $x_{5}$ is internal. According to Proposition 3 with $y=x_{5}$ we can remove the last row of the RHS matrix, substitute $x_{2}$ for $x_{5}$ everywhere else and forget about $x_{5}$. We end up with the Koszul matrix of $C\left(\Gamma_{2}\right)$.

Proof of Proposition 1 To show independence of $C(D)$ on the number and position of marks, we need to check compatibility of the isomorphisms $C\left(\Gamma_{1}\right) \cong C\left(\Gamma_{2}\right)$ above with maps $\chi_{0}, \chi_{1}$. We will only work through one case and leave the others to an interested reader. Let's check that complexes of factorizations

$$
0 \longrightarrow C\left(\Gamma_{1}^{1}\right) \stackrel{\chi_{1}}{\longrightarrow} C\left(\Gamma_{1}^{0}\right) \longrightarrow 0
$$

and

$$
0 \longrightarrow C\left(\Gamma_{2}^{1}\right) \stackrel{\chi_{1}}{\longrightarrow} C\left(\Gamma_{2}^{0}\right) \longrightarrow 0
$$



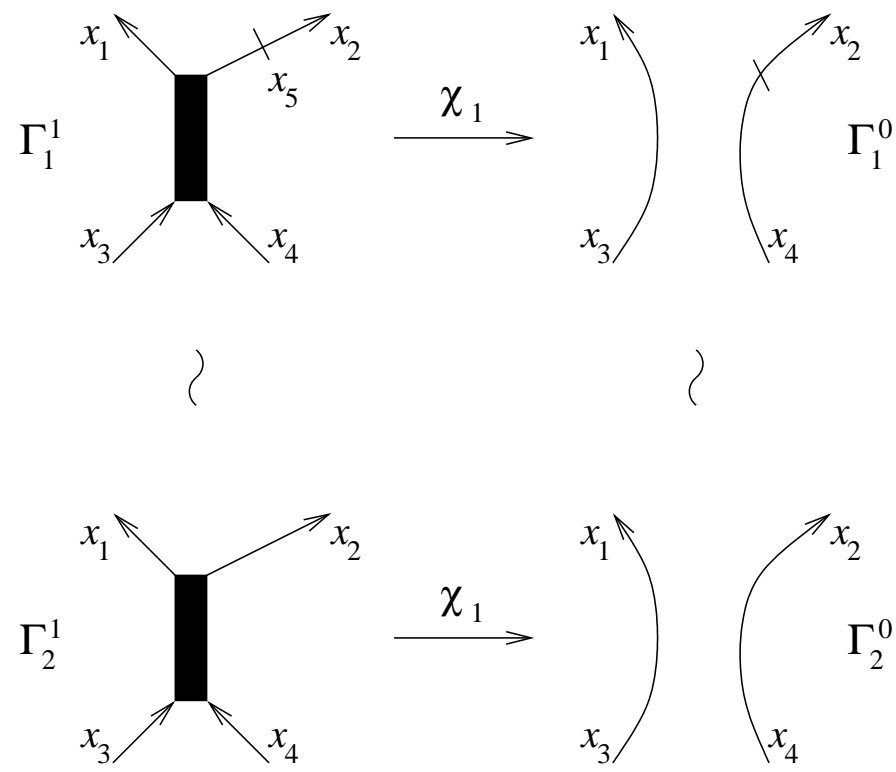

Figure 10

are chain homotopy equivalent, for Figure 10 diagrams. The first complex, written via Koszul matrices, has the form

$$
\left(\begin{array}{cc}
a & x_{1}+x_{5}-x_{3}-x_{4} \\
0 & \left(x_{5}-x_{3}\right)\left(x_{4}-x_{5}\right) \\
a & x_{2}-x_{5}
\end{array}\right) \stackrel{\operatorname{Id} \otimes \psi\left(x_{4}-x_{5}\right) \otimes \operatorname{Id}}{\longrightarrow}\left(\begin{array}{cc}
a & x_{1}+x_{5}-x_{3}-x_{4} \\
0 & x_{5}-x_{3} \\
a & x_{2}-x_{5}
\end{array}\right)
$$

Applying $[13]_{1}$ simultaneously to both matrices we get an isomorphic complex

$$
\left(\begin{array}{cc}
a & x_{1}+x_{2}-x_{3}-x_{4} \\
0 & \left(x_{5}-x_{3}\right)\left(x_{4}-x_{5}\right) \\
0 & x_{2}-x_{5}
\end{array}\right) \stackrel{\operatorname{Id} \otimes \psi\left(x_{4}-x_{5}\right) \otimes \operatorname{Id}}{\longrightarrow}\left(\begin{array}{cc}
a & x_{1}+x_{5}-x_{3}-x_{4} \\
0 & x_{5}-x_{3} \\
0 & x_{2}-x_{5}
\end{array}\right) \text {. }
$$

The only internal variable is $x_{5}$. We switch from $x_{5}$ to $x=x_{2}-x_{5}$. We think of $x$ as an internal variable, while $a, x_{1}, x_{2}, x_{3}, x_{4}$ are external. Both matrices have identical bottom rows $(0, x)$ and the differential is the identity on that row. Therefore, we can eliminate $x$ from the complex, reducing the ground ring to $\mathbb{Q}\left[a, x_{1}, x_{2}, x_{3}, x_{4}\right]$, crossing out the bottom row and setting $x=0$. The resulting complex

$$
\left(\begin{array}{cc}
a & x_{1}+x_{2}-x_{3}-x_{4} \\
0 & \left(x_{2}-x_{3}\right)\left(x_{4}-x_{2}\right)
\end{array}\right) \stackrel{\operatorname{Id} \otimes \psi\left(x_{4}-x_{2}\right)}{\longrightarrow}\left(\begin{array}{cc}
a & x_{1}+x_{2}-x_{3}-x_{4} \\
0 & x_{2}-x_{3}
\end{array}\right)
$$


is isomorphic to (23).

\subsection{Invariance under Reidemeister move I}

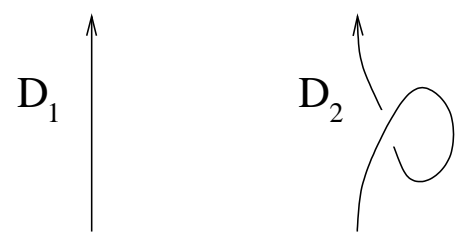

Figure 11: Type IA Reidemeister move

Consider a type IA Reidemeister move as depicted in Figure 11. The complex $C\left(D_{2}\right)\{0,2\}$ has the form

$$
\left(0 \longrightarrow C\left(\Gamma_{1}\right)_{-\frac{1}{2}} \stackrel{\chi_{1}}{\longrightarrow} C\left(\Gamma_{0}\right)_{\frac{1}{2}} \longrightarrow 0\right)\left\{\frac{1}{2},-\frac{3}{2}\right\},
$$

see Figure 12. In terms of Koszul matrices, the complex is given by

$$
\left(\begin{array}{cc}
a & x_{1}-x_{4} \\
0 & 0
\end{array}\right) \stackrel{\operatorname{Id} \otimes \psi\left(x_{4}-x_{2}\right)}{\longrightarrow}\left(\begin{array}{cc}
a & x_{1}-x_{4} \\
0 & 0
\end{array}\right)
$$

(we set $x_{3}=x_{2}$ in the formulas (21), (22) for $\Gamma^{0}$ and $\Gamma^{1}$ ).
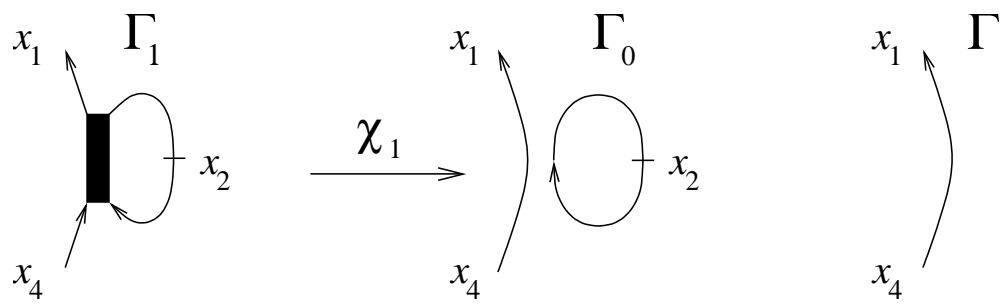

Figure 12: Resolutions of $D_{2}$

The differential is the identity on the first row, and on the second row given by

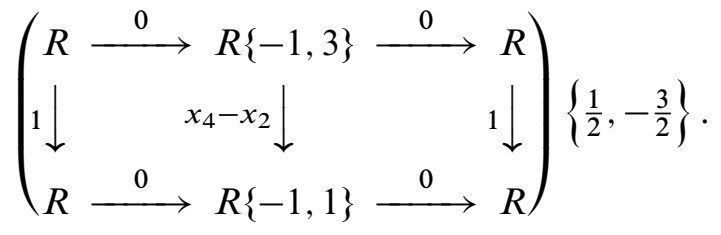

Geometry 83 Topology, Volume 12 (2008) 
Therefore the complex splits into a direct sum of a contractible complex and the tensor product of $\left(a, x_{1}-x_{4}\right)$ with

$$
0 \longrightarrow R_{-\frac{1}{2}}\left\{-\frac{1}{2}, \frac{3}{2}\right\} \stackrel{x_{4}-x_{2}}{\longrightarrow} R_{\frac{1}{2}}\left\{-\frac{1}{2},-\frac{1}{2}\right\} \longrightarrow 0 .
$$

Since $x_{2}$ is an internal variable, we can remove a contractible summand

$$
0 \longrightarrow R_{-\frac{1}{2}}\left\{-\frac{1}{2}, \frac{3}{2}\right\} \stackrel{1}{\rightarrow} R\left(x_{4}-x_{2}\right)_{\frac{1}{2}}\left\{-\frac{1}{2},-\frac{1}{2}\right\} \longrightarrow 0
$$

from the above complex and reduce the ground ring to $R^{\prime}=\mathbb{Q}\left[a, x_{1}, x_{4}\right]$. We get the complex $\left(a, x_{1}-x_{4}\right)$ shifted by $\left\{-\frac{1}{2},-\frac{1}{2}\right\}\left[-\frac{1}{2}\right]$. Thus,

$$
C\left(D_{2}\right)\left\{\frac{1}{2}, \frac{1}{2}\right\}\left[\frac{1}{2}\right] \simeq C(\Gamma)
$$

for $\Gamma$ as in Figure 12. Since $\Gamma \cong D_{1}$, we arrive at equation (20). We record this as the following proposition.

Proposition 4 Complexes of matrix factorizations $C\left(D_{1}\right)$ and $C\left(D_{2}\right)\left\{\frac{1}{2}, \frac{1}{2}\right\}\left[\frac{1}{2}\right]$ are isomorphic as objects of $K\left(h m f_{w}\right)$, with $w=a\left(x_{1}-x_{4}\right)$.

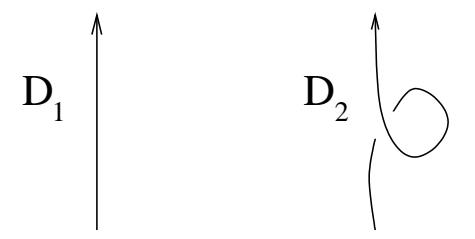

Figure 13: Type IB Reidemeister move

A similar computation takes care of the Reidemeister move IB.

Proposition 5 Complexes of matrix factorizations $C\left(D_{1}\right)$ and $C\left(D_{2}\right)\left\{\frac{1}{2}, \frac{1}{2}\right\}\left[\frac{1}{2}\right]$ are isomorphic as objects of $K\left(h m f_{w}\right)$, for $D_{1}, D_{2}$ depicted in Figure 13.

\subsection{Invariance under Reidemeister move IIa}

Complexes of matrix factorizations $C\left(D_{1}\right)$ and $C\left(D_{2}\right)$, for the diagrams depicted in Figure 14, live in the category $K\left(h m f_{w}\right)$ with $w=a\left(x_{1}+x_{2}-x_{3}-x_{4}\right)$, viewed as an element of the ground ring $R=\mathbb{Q}\left[a, x_{1}, x_{2}, x_{3}, x_{4}\right]$. The complex $C\left(D_{2}\right) \cong C\left(\Gamma_{01}\right)$ lies entirely in cohomological degree zero, since $D_{2}$ has no crossings. 

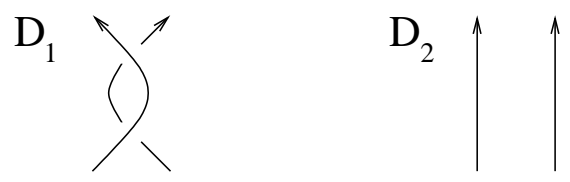

Figure 14: Type IIa move
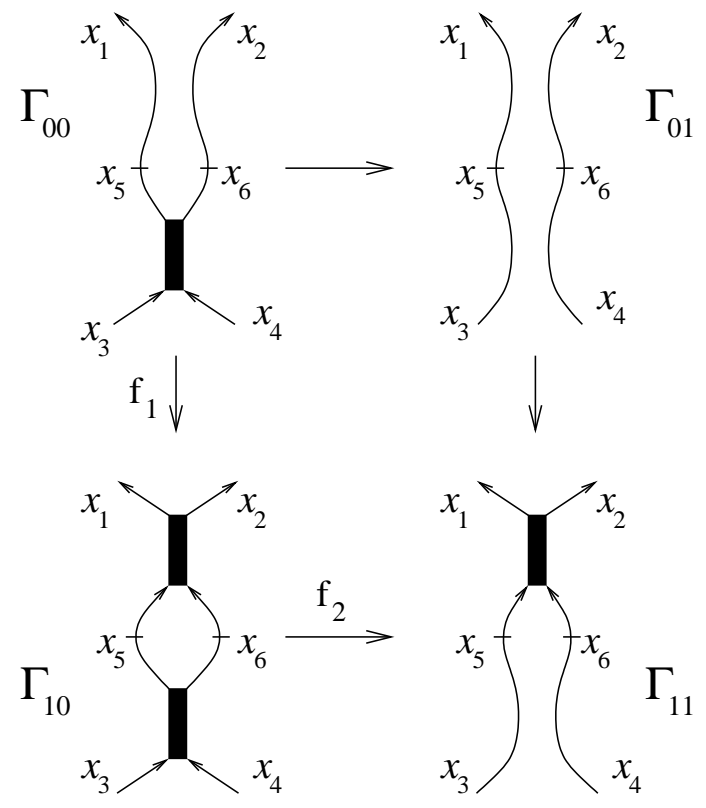

Figure 15: Four resolutions of $D_{1}$

Proposition 6 Complexes of matrix factorizations $C\left(D_{1}\right)$ and $C\left(D_{2}\right)$ are equivalent as objects of $K\left(h m f_{w}\right)$.

Proof It suffices to show that $f_{1}$ is an isomorphism (in $h m f_{w}$ ) from $C\left(\Gamma_{00}\right)$ to a direct summand of $C\left(\Gamma_{10}\right)$, and that there is a decomposition

$$
C\left(\Gamma_{10}\right) \cong \operatorname{Im}(f) \oplus M
$$


where $M$ is isomorphic to $C\left(\Gamma_{11}\right)$, with $\left.f_{2}\right|_{M}$ establishing this isomorphism. Then $C\left(D_{1}\right)$ would be isomorphic to a direct sum of contractible complexes

$$
\begin{aligned}
& 0 \longrightarrow C\left(\Gamma_{00}\right) \stackrel{\mathrm{Id}}{\longrightarrow} \operatorname{Im}(f) \longrightarrow 0, \\
& 0 \longrightarrow M \stackrel{f_{2}}{\longrightarrow} C\left(\Gamma_{11}\right) \longrightarrow 0
\end{aligned}
$$

and the factorization $C\left(\Gamma_{01}\right)$, isomorphic to $C\left(D_{2}\right)$. Since the matrix factorizations $C\left(\Gamma_{0}\right)$ in the complexes (12) and (13) have opposite degree shifts, the matrix factorization $C\left(D_{2}\right)$ in Figure 15 has zero degree shift. Thus the homotopy equivalence between the complexes $C\left(D_{1}\right)$ and $C\left(D_{2}\right)$ would be established.

We start by writing down the diagram of factorizations and maps

$$
C\left(\Gamma_{00}\right) \stackrel{f_{1}}{\longrightarrow} C\left(\Gamma_{10}\right) \stackrel{f_{2}}{\longrightarrow} C\left(\Gamma_{11}\right)
$$

and simplify them in $h m f_{w}$ by removing contractible direct summand factorizations from each of the three terms. The diagram has the form

$$
\begin{aligned}
& \left(\begin{array}{cc}
a & x_{1}+x_{2}-x_{5}-x_{6} \\
0 & x_{2}-x_{6} \\
a & x_{5}+x_{6}-x_{3}-x_{4} \\
0 & \left(x_{6}-x_{4}\right)\left(x_{3}-x_{6}\right)
\end{array}\right) \stackrel{f_{1}}{\longrightarrow}\left(\begin{array}{cc}
a & x_{1}+x_{2}-x_{5}-x_{6} \\
0 & \left(x_{2}-x_{6}\right)\left(x_{5}-x_{2}\right) \\
a & x_{5}+x_{6}-x_{3}-x_{4} \\
0 & \left(x_{6}-x_{4}\right)\left(x_{3}-x_{6}\right)
\end{array}\right) \stackrel{f_{2}}{\longrightarrow} \\
& \left(\begin{array}{lc}
a & x_{1}+x_{2}-x_{5}-x_{6} \\
0 & \left(x_{2}-x_{6}\right)\left(x_{5}-x_{2}\right) \\
a & x_{5}+x_{6}-x_{3}-x_{4} \\
0 & x_{6}-x_{4}
\end{array}\right)
\end{aligned}
$$

with

$$
f_{1}=\mathrm{Id} \otimes \psi^{\prime}\left(x_{5}-x_{2}\right) \otimes \mathrm{Id}^{\otimes 2}, \quad f_{2}=\mathrm{Id}^{\otimes 3} \otimes \psi\left(x_{3}-x_{6}\right) .
$$

Apply row transformation $[13]_{1}$ to all three Koszul matrices. The new matrices will have identical first rows $\left(a, x_{1}+x_{2}-x_{3}-x_{4}\right)$ and identical third rows $\left(0, x_{5}+x_{6}-x_{3}-x_{4}\right)$. We remove the third rows and exclude internal variable $x_{6}$ substituting $x_{3}+x_{4}-x_{5}$ in its place everywhere else. The diagram becomes the tensor product of the Koszul factorization $\left(a, x_{1}+x_{2}-x_{3}-x_{4}\right)$ and the diagram

$$
\begin{aligned}
& \left(\begin{array}{c}
x_{2}-x_{6} \\
\left(x_{6}-x_{4}\right)\left(x_{3}-x_{6}\right)
\end{array}\right) \stackrel{g_{1}}{\longrightarrow}\left(\begin{array}{c}
\left(x_{2}-x_{6}\right)\left(x_{3}+x_{4}-x_{6}-x_{2}\right) \\
\left(x_{6}-x_{4}\right)\left(x_{3}-x_{6}\right)
\end{array}\right) \stackrel{g_{2}}{\longrightarrow} \\
& \left(\begin{array}{c}
\left(x_{2}-x_{6}\right)\left(x_{3}+x_{4}-x_{6}-x_{2}\right) \\
x_{6}-x_{4}
\end{array}\right)
\end{aligned}
$$


where

$$
g_{1}=\psi^{\prime}\left(x_{3}+x_{4}-x_{6}-x_{2}\right) \otimes \mathrm{Id}, \quad g_{2}=\mathrm{Id} \otimes \psi\left(x_{3}-x_{6}\right) .
$$

We omitted the first columns from the Koszul matrices, since their terms are all zeros. The only internal variable left is $x_{6}$. The bottom term in the first two factorizations is

$$
\left(x_{6}-x_{4}\right)\left(x_{3}-x_{6}\right)=-x_{6}^{2}+\left(x_{3}+x_{4}\right) x_{6}-x_{3} x_{4} .
$$

Let $R^{\prime}=\mathbb{Q}\left[a, x_{1}, x_{2}, x_{3}, x_{4}\right]$ be the polynomial ring on all external variables. Currently we are working over the ring $R^{\prime}\left[x_{6}\right]$. We remove the bottom term from the first two factorizations simultaneously reducing to $R^{\prime}$, imposing the relation $x_{6}^{2}=\left(x_{3}+x_{4}\right) x_{6}-$ $x_{3} x_{4}$, and treating multiplication by $x_{6}$ as an endomorphism of the free $R^{\prime}$-module $R^{\prime}\left[x_{6}\right] /\left(\left(x_{6}-x_{4}\right)\left(x_{3}-x_{6}\right)\right)$. Likewise, in the rightmost factorization, we remove the bottom row $\left(x_{6}-x_{4}\right)$, reduce to the ground ring $R^{\prime}$ and impose the relation $x_{6}=x_{4}$. Our diagram simplifies to

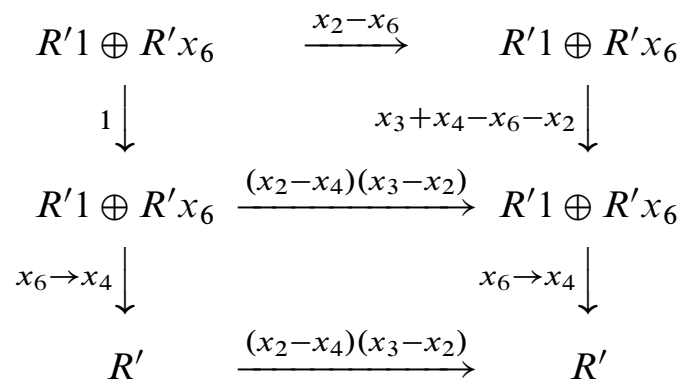

where, for instance, the bottom row denotes the factorization

$$
R^{\prime} \stackrel{0}{\longrightarrow} R^{\prime} \stackrel{\left(x_{2}-x_{4}\right)\left(x_{3}-x_{2}\right)}{\longrightarrow} R^{\prime}
$$

and the maps $g_{1}, g_{2}$ are given by vertical arrows. Stripping off a contractible summand

$$
R^{\prime} 1 \stackrel{1}{\longrightarrow} R^{\prime}\left(x_{2}-x_{6}\right)
$$

from the first factorization, we reduce it to

$$
R^{\prime}\left(x_{6}+x_{2}-x_{3}-x_{4}\right) \stackrel{\left(x_{2}-x_{4}\right)\left(x_{2}-x_{3}\right)}{\longrightarrow} R^{\prime} 1 .
$$

The middle factorization is a direct sum of two isomorphic (up to grading shift) factorizations

$$
R^{\prime} 1 \stackrel{\left(x_{2}-x_{4}\right)\left(x_{3}-x_{2}\right)}{\longrightarrow} R^{\prime} 1
$$

and

$$
R^{\prime}\left(x_{6}+x_{2}-x_{3}-x_{4}\right) \stackrel{\left(x_{2}-x_{4}\right)\left(x_{3}-x_{2}\right)}{\longrightarrow} R^{\prime}\left(x_{6}+x_{2}-x_{3}-x_{4}\right) .
$$


The map $g_{1}$ takes the top factorization (in its reduced form) isomorphically onto the second summand of the middle factorization. The map $g_{2}$ restricts to an isomorphism from the first direct summand of the middle factorization to the bottom factorization. Our claim and the proposition follow.

The invariance under the mirror image of the Figure 14 move can be verified similarly.

\subsection{Invariance under Reidemeister move III}

Let factorization $\Upsilon$ be given by the following Koszul matrix:

$$
\left(\begin{array}{cc}
a & x_{1}+x_{2}+x_{3}-x_{4}-x_{5}-x_{6} \\
0 & x_{1} x_{2}+x_{1} x_{3}+x_{2} x_{3}-x_{4} x_{5}-x_{4} x_{6}-x_{5} x_{6} \\
0 & x_{1} x_{2} x_{3}-x_{4} x_{5} x_{6}
\end{array}\right) .
$$

The gradings are normalized so that the differential has bidegree $(1,1)$. For instance, the bottom row denotes the factorization

$$
R \stackrel{0}{\longrightarrow} R\{-1,5\} \stackrel{x_{1} x_{2} x_{3}-x_{4} x_{5} x_{6}}{\longrightarrow} R
$$

with $R=\mathbb{Q}\left[a, x_{1}, \ldots, x_{6}\right]$. The potential is $w=a\left(x_{1}+x_{2}+x_{3}-x_{4}-x_{5}-x_{6}\right)$.
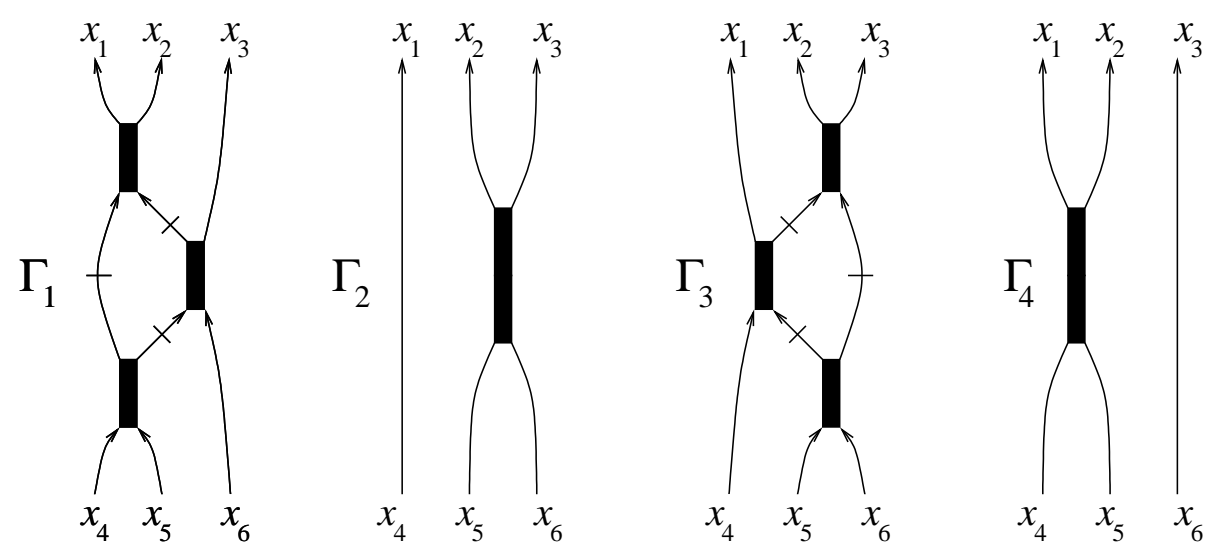

Figure 16: Diagrams $\Gamma_{1}, \Gamma_{2}, \Gamma_{3}, \Gamma_{4}$

Proposition 7 In $h m f_{w}$ there are isomorphisms

$$
\begin{aligned}
& C\left(\Gamma_{1}\right) \cong C\left(\Gamma_{4}\right)\{0,2\} \oplus \Upsilon, \\
& C\left(\Gamma_{3}\right) \cong C\left(\Gamma_{2}\right)\{0,2\} \oplus \Upsilon,
\end{aligned}
$$

for $\Gamma_{1}, \Gamma_{2}, \Gamma_{3}, \Gamma_{4}$ depicted in Figure 16 . 
Proof To prove the first isomorphism, we place labels $x_{7}, x_{8}, x_{9}$ (from top to bottom) on the three marks of $\Gamma_{1}$ and write $C\left(\Gamma_{1}\right)$ in the Koszul form

$$
C\left(\Gamma_{1}\right)=\left(\begin{array}{cc}
a & x_{1}+x_{2}-x_{8}-x_{7} \\
0 & \left(x_{2}-x_{7}\right)\left(x_{8}-x_{2}\right) \\
a & x_{7}+x_{3}-x_{9}-x_{6} \\
0 & \left(x_{3}-x_{6}\right)\left(x_{9}-x_{3}\right) \\
a & x_{8}+x_{9}-x_{4}-x_{5} \\
0 & \left(x_{9}-x_{5}\right)\left(x_{4}-x_{9}\right)
\end{array}\right)
$$

Applying transformations $[13]_{1}$ and $[15]_{1}$ we get a matrix with the third and fifth rows

$$
\left(0, x_{7}+x_{3}-x_{9}-x_{6}\right), \quad\left(0, x_{8}+x_{9}-x_{4}-x_{5}\right) .
$$

We use these rows to exclude internal variables $x_{7}$ and $x_{8}$ and reduce $C\left(\Gamma_{1}\right)$ to the following Koszul form

$$
\left(\begin{array}{cc}
a & x_{1}+x_{2}+x_{3}-x_{4}-x_{5}-x_{6} \\
0 & \left(x_{2}+x_{3}-x_{6}-x_{9}\right)\left(x_{4}+x_{5}-x_{2}-x_{9}\right) \\
0 & \left(x_{3}-x_{6}\right)\left(x_{9}-x_{3}\right) \\
0 & \left(x_{9}-x_{5}\right)\left(x_{4}-x_{9}\right)
\end{array}\right)
$$

Notice that variables $a$ and $x_{1}$ appear only in the first row. Moreover, Koszul forms of factorizations $\Upsilon$ and $C\left(\Gamma_{4}\right)$ have the same first row. Next, we ignore the first row of $C\left(\Gamma_{1}\right)$ and operate on the other three rows. The first column of the Koszul matrix then consists of zeros and we omit it. To simplify the factorization

$$
\left(\begin{array}{c}
\left(x_{2}+x_{3}-x_{6}-x_{9}\right)\left(x_{4}+x_{5}-x_{2}-x_{9}\right) \\
\left(x_{3}-x_{6}\right)\left(x_{9}-x_{3}\right) \\
\left(x_{9}-x_{5}\right)\left(x_{4}-x_{9}\right)
\end{array}\right)
$$

we use the last term to reduce to at most linear terms in the last remaining internal variable $x_{9}$. Remove the last row and impose the relation $x_{9}^{2}=\left(x_{4}+x_{5}\right) x_{9}-x_{4} x_{5}$. Modulo this relation and after adding the second row, the first row loses $x_{9}$ and the matrix becomes

$$
\left(\begin{array}{c}
\left(x_{3}-x_{6}\right)\left(x_{4}+x_{5}-x_{2}-x_{3}\right)+\left(x_{2}-x_{5}\right)\left(x_{4}-x_{2}\right) \\
\left(x_{3}-x_{6}\right)\left(x_{9}-x_{3}\right)
\end{array}\right) .
$$

Now $x_{9}$ appears only in the bottom row, which we can write as

$$
R 1 \oplus R x_{9} \stackrel{\left(x_{3}-x_{6}\right)\left(x_{9}-x_{3}\right)}{\longrightarrow} R 1 \oplus R x_{9} .
$$

Changing basis of the free $R$-module on the left hand side from $\left\{1, x_{9}\right\}$ to $\left\{1, x_{9}+\right.$ $\left.x_{3}-x_{4}-x_{5}\right\}$ and of the module on the right to $\left\{1, x_{9}-x_{3}\right\}$, we decompose this 
complex into a direct sum of

$$
R\left(x_{9}+x_{3}-x_{4}-x_{5}\right) \stackrel{\left(x_{3}-x_{4}\right)\left(x_{5}-x_{3}\right)\left(x_{3}-x_{6}\right)}{\longrightarrow} R 1
$$

and

$$
R 1 \stackrel{x_{3}-x_{6}}{\longrightarrow} R\left(x_{9}-x_{3}\right)
$$

Adding the other rows, we obtain a decomposition of $C\left(\Gamma_{1}\right)$ into direct sum of factorizations with Koszul matrices

$$
\left(\begin{array}{cc}
a & x_{1}+x_{2}+x_{3}-x_{4}-x_{5}-x_{6} \\
0 & \left(x_{3}-x_{6}\right)\left(x_{4}+x_{5}-x_{2}-x_{3}\right)+\left(x_{2}-x_{5}\right)\left(x_{4}-x_{2}\right) \\
0 & \left(x_{3}-x_{4}\right)\left(x_{3}-x_{5}\right)\left(x_{3}-x_{6}\right)
\end{array}\right)
$$

and

$$
\left(\begin{array}{cc}
a & x_{1}+x_{2}+x_{3}-x_{4}-x_{5}-x_{6} \\
0 & \left(x_{3}-x_{6}\right)\left(x_{4}+x_{5}-x_{2}-x_{3}\right)+\left(x_{2}-x_{5}\right)\left(x_{4}-x_{2}\right) \\
0 & x_{3}-x_{6}
\end{array}\right),
$$

the latter shifted by $\{0,2\}$ due to the bidegree $(0,2)$ vector $x_{9}-x_{3}$ being a generator of the module $R\left(x_{9}-x_{3}\right)$. It is easy to check that the matrices above describe factorizations $\Upsilon$ and $C\left(\Gamma_{4}\right)$, respectively.

Proposition 8 Complexes $C\left(D_{1}\right)$ and $C\left(D_{2}\right)$, for diagrams depicted in Figure 17, are isomorphic in the category $K\left(h m f_{w}\right)$.
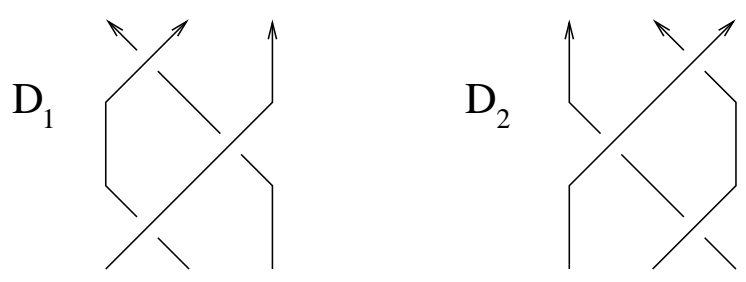

Figure 17: Reidemeister move III

Proof The proof is similar to the one in [8]. The complex $C\left(D_{1}\right)$ consists of eight factorizations assigned to diagrams depicted in Figure 18 (also see Figure 6). We ignore the overall shift by $\left\{\frac{1}{2},-\frac{3}{2}\right\}$ in the resolution of each crossing, which was needed for the invariance under the Reidemeister move IIa, but does not make any difference for Reidemeister move III. Proposition 7 tells us that

$$
C\left(\Gamma_{111}\right) \cong \Upsilon \oplus C\left(\Gamma_{100}\right)\{0,2\}
$$


(also observe that $\Gamma_{100} \cong \Gamma_{001}$ ), while our proof of the invariance under the Reidemeister move IIa implies

$$
C\left(\Gamma_{101}\right) \cong C\left(\Gamma_{100}\right)\{0,2\} \oplus C\left(\Gamma_{100}\right)
$$

A computation similar to the one in that proof shows that the map $\chi_{1}: C\left(\Gamma_{111}\right) \longrightarrow$ $C\left(\Gamma_{101}\right)$, when restricted to the direct summand isomorphic to $C\left(\Gamma_{100}\right)\{0,2\}$, is an isomorphism onto a direct summand of $C\left(\Gamma_{101}\right)$, while our proof of Proposition 6 implies that $\chi_{1}: C\left(\Gamma_{101}\right) \longrightarrow C\left(\Gamma_{001}\right)$ is an isomorphism when restricted to the direct summand $C\left(\Gamma_{100}\right)$ of $C\left(\Gamma_{101}\right)$.

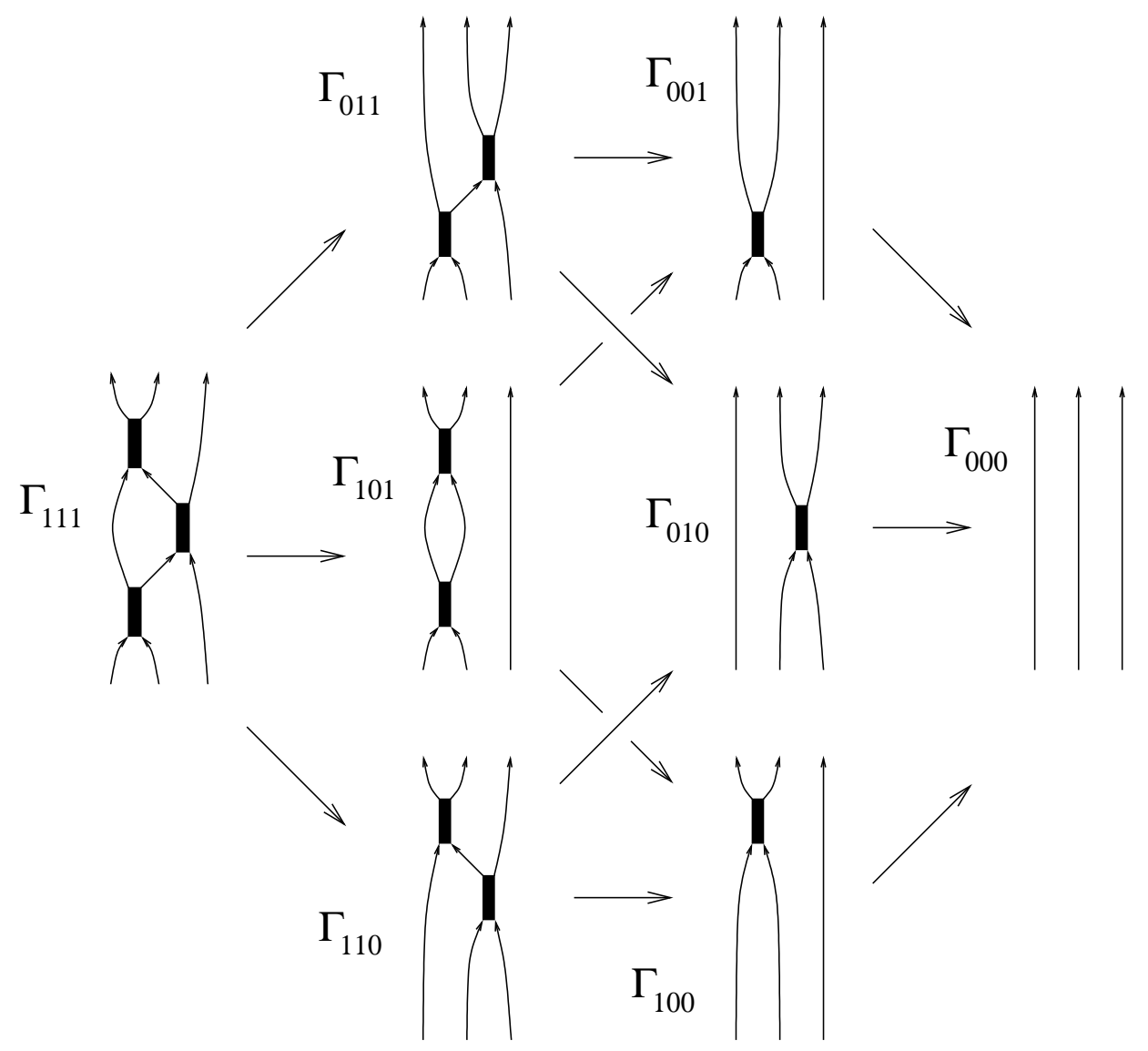

Figure 18: Resolution cube of $D_{1}$ 
After removing contractible summands

$$
0 \longrightarrow C\left(\Gamma_{100}\right)\{0,2\} \stackrel{\cong}{\longrightarrow} C\left(\Gamma_{100}\right)\{0,2\} \longrightarrow 0
$$

and

$$
0 \longrightarrow C\left(\Gamma_{100}\right) \stackrel{\cong}{\longrightarrow} C\left(\Gamma_{100}\right) \longrightarrow 0
$$

we reduce $C\left(D_{1}\right)$ to a complex $C^{\prime}$ that is graphically depicted in Figure 19, where factorization $\Upsilon$ assigned to the diagram $Y$.

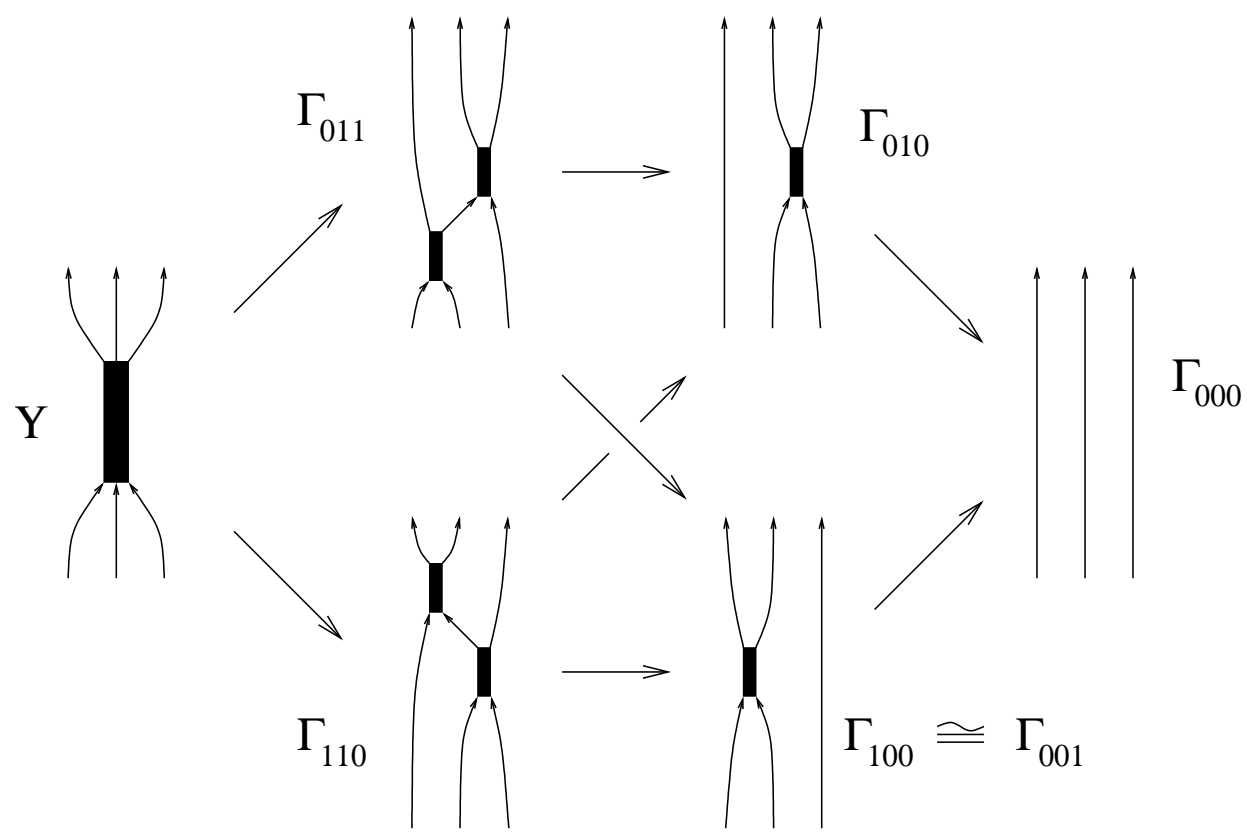

Figure 19: Complex $C^{\prime}$

Lemma 4 The complex $C^{\prime} \cong C\left(D_{1}\right)$ is indecomposable in the category $K\left(h m f_{w}\right)$.

Proof In other words, we cannot write $C^{\prime} \cong M \oplus N$ for two nontrivial objects $M, N$ of $K\left(h m f_{w}\right)$. Indeed, invariance under the Reidemeister move IIa tells us that tensoring with a complex of factorizations assigned to a crossing is an invertible functor. Precisely, it is an invertible functor from the category $K\left(h m f_{v}\right)$ to $K\left(h m f_{u}\right)$ where $v=a x_{3}+a x_{4}+f(\mathbf{x}), u=a x_{1}+a x_{2}+f(\mathbf{x})$, and $f(\mathbf{x})$ is any polynomial in variables $\mathbf{x}$ disjoint from $x_{1}, \ldots, x_{4}$. An invertible functor is indecomposable iff the identity functor is. The identity functor, in general, corresponds to the diagram comprised of $n$ 
parallel lines, compatibly oriented (the diagram of the trivial braid). Its factorization $S$ can be written as the tensor product of $\left(a, x_{i}-x_{n+i}\right)$, over $i=1, \ldots, n$. An easy computation (for instance, as in the proof of the next lemma) shows that the hom space $\operatorname{Hom}_{h m f_{w}}(S, S)$ of bigrading-preserving factorization homomorphisms up to chain homotopies is one-dimensional. Therefore, $S$ and the identity functor are indecomposable, for otherwise a projection onto a direct summand would ensure that the above hom space is at least 2-dimensional.

Lemma 5 For any arrow $\Gamma \longrightarrow \Gamma^{\prime}$ in Figure 19, the space of bidegree-preserving maps $C(\Gamma) \rightarrow C\left(\Gamma^{\prime}\right)$ is one-dimensional (over the ground field $\mathbb{Q}$ ) and is generated by $\chi_{1}$.

Proof We can prove the lemma on a case-by-case basis, separately for each arrow. In general, to compute the dimension of $\operatorname{Hom}_{h m f_{w}}(M, N)$, for matrix factorizations $M, N$, with $M$ of finite rank, we use the isomorphism

$$
\operatorname{EXT}_{h m f_{w}}(M, N) \cong H\left(N \otimes_{R} M_{\bullet}\right)
$$

where EXT refers to taking ext groups of the pair $M, N$ in all bidegrees, $M_{\bullet}$ is the $R$-module dual of $M$, and $H$ stands for cohomology. Restricting the left hand side to Hom corresponds to taking the bidegree $(0,0)$ summand of the right hand side. The dual of a Koszul factorization $\left(a_{i}, b_{i}\right)$ is the Koszul factorization $\left(b_{i},-a_{i}\right)$, with suitably shifted gradings.

For instance, to determine the dimension of the space

$$
\operatorname{Hom}_{h m f_{w}}\left(C\left(\Gamma_{110}\right), C\left(\Gamma_{100}\right)\right)
$$

(the bottom arrow in Figure 19) we first write the Koszul matrix of $C\left(\Gamma_{110}\right)$ :

$$
\left(\begin{array}{llll}
\{0,0\} & a & \{1,-1\} & x_{1}+x_{2}-x_{4}-x_{7} \\
\{0,0\} & a & \{1,-1\} & x_{7}+x_{3}-x_{5}-x_{6} \\
\{0,0\} & 0 & \{1,-3\} & \left(x_{2}-x_{4}\right)\left(x_{2}-x_{7}\right) \\
\{0,0\} & 0 & \{1,-3\} & \left(x_{3}-x_{5}\right)\left(x_{3}-x_{6}\right)
\end{array}\right)
$$

Here $x_{7}$ is the variable assigned to the internal mark of $\Gamma_{110}$. We also added two columns indicating the bidegrees of $R$. For instance, the second row denotes the factorization

$$
R\{0,0\} \stackrel{a}{\longrightarrow} R\{1,-1\} \stackrel{x_{7}+x_{3}-x_{5}-x_{6}}{\longrightarrow} R\{0,0\} .
$$

After we apply $[12]_{1}$, the second row becomes $\left(0, x_{7}+x_{3}-x_{5}-x_{6}\right)$; we get rid of it and of the variable $x_{7}$. Thus, $C\left(\Gamma_{110}\right)$ is isomorphic to the factorization assigned to 
the Koszul matrix

$$
\left(\begin{array}{cccc}
\{0,0\} & a & \{-1,1\} & x_{1}+x_{2}+x_{3}-x_{4}-x_{5}-x_{6} \\
\{0,0\} & 0 & \{-1,3\} & \left(x_{2}-x_{4}\right)\left(x_{2}+x_{3}-x_{5}-x_{6}\right) \\
\{0,0\} & 0 & \{-1,3\} & \left(x_{3}-x_{5}\right)\left(x_{3}-x_{6}\right)
\end{array}\right) .
$$

The dual $C\left(\Gamma_{110}\right) \bullet$ of $C\left(\Gamma_{110}\right)$ can be represented by the matrix

$$
\left(\begin{array}{cccc}
\{0,0\} & x_{1}+x_{2}+x_{3}-x_{4}-x_{5}-x_{6} & \{1,-1\} & -a \\
\{0,0\} & \left(x_{2}-x_{4}\right)\left(x_{2}+x_{3}-x_{5}-x_{6}\right) & \{1,-3\} & 0 \\
\{0,0\} & \left(x_{3}-x_{5}\right)\left(x_{3}-x_{6}\right) & \{1,-3\} & 0
\end{array}\right)
$$

and the tensor product complex $C\left(\Gamma_{110}\right) \bullet \otimes C\left(\Gamma_{100}\right)$ by

$$
\left(\begin{array}{cccc}
\{0,0\} & a & \{-1,1\} & x_{1}+x_{2}-x_{4}-x_{5} \\
\{0,0\} & 0 & \{-1,3\} & \left(x_{2}-x_{4}\right)\left(x_{2}-x_{5}\right) \\
\{0,0\} & a & \{-1,1\} & x_{3}-x_{6} \\
\{0,0\} & x_{1}+x_{2}+x_{3}-x_{4}-x_{5}-x_{6} & \{1,-1\} & -a \\
\{0,0\} & \left(x_{2}-x_{4}\right)\left(x_{2}+x_{3}-x_{5}-x_{6}\right) & \{1,-3\} & 0 \\
\{0,0\} & \left(x_{3}-x_{5}\right)\left(x_{3}-x_{6}\right) & \{1,-3\} & 0
\end{array}\right),
$$

where the first three rows describe $C\left(\Gamma_{100}\right)$. We do transformation $[13]_{1}$ and shift rows 4 and 5 by one each. We get

$$
\left(\begin{array}{cccc}
\{0,0\} & a & \{-1,1\} & x_{1}+x_{2}+x_{3}-x_{4}-x_{5}-x_{6} \\
\{0,0\} & 0 & \{-1,3\} & \left(x_{2}-x_{4}\right)\left(x_{2}-x_{5}\right) \\
\{0,0\} & 0 & \{-1,1\} & x_{3}-x_{6} \\
\{1,-1\} & -a & \{0,0\} & x_{1}+x_{2}+x_{3}-x_{4}-x_{5}-x_{6} \\
\{1,-3\} & 0 & \{0,0\} & \left(x_{2}-x_{4}\right)\left(x_{2}+x_{3}-x_{5}-x_{6}\right) \\
\{0,0\} & \left(x_{3}-x_{5}\right)\left(x_{3}-x_{6}\right) & \{1,-3\} & 0
\end{array}\right) .
$$

We apply the transformation $[14]_{-1}$, then shift rows 1 and 6 to obtain

$$
\left(\begin{array}{cccc}
\{-1,1\} & 0 & \{0,0\} & a \\
\{0,0\} & 0 & \{-1,3\} & \left(x_{2}-x_{4}\right)\left(x_{2}-x_{5}\right) \\
\{0,0\} & 0 & \{-1,1\} & x_{3}-x_{6} \\
\{1,-1\} & 0 & \{0,0\} & x_{1}+x_{2}+x_{3}-x_{4}-x_{5}-x_{6} \\
\{1,-3\} & 0 & \{0,0\} & \left(x_{2}-x_{4}\right)\left(x_{2}+x_{3}-x_{5}-x_{6}\right) \\
\{1,-3\} & 0 & \{0,0\} & \left(x_{3}-x_{5}\right)\left(x_{3}-x_{6}\right)
\end{array}\right)
$$


Cross out row 3 and convert $x_{6}$ to $x_{3}$ everywhere else. The matrix reduces to

$$
\left(\begin{array}{cccc}
\{-1,1\} & 0 & \{0,0\} & a \\
\{0,0\} & 0 & \{-1,3\} & \left(x_{2}-x_{4}\right)\left(x_{2}-x_{5}\right) \\
\{1,-1\} & 0 & \{0,0\} & x_{1}+x_{2}-x_{4}-x_{5} \\
\{1,-3\} & 0 & \{0,0\} & \left(x_{2}-x_{4}\right)\left(x_{2}-x_{5}\right) \\
\{1,-3\} & 0 & \{0,0\} & 0
\end{array}\right)
$$

We apply [24] $]_{1}$, then remove rows 1 and 3 simultaneously with getting rid of the variables $a$ and $x_{5}$. The resulting matrix is

$$
\left(\begin{array}{cccc}
\{0,0\} & 0 & \{-1,3\} & \left(x_{2}-x_{4}\right)\left(x_{4}-x_{1}\right) \\
\{1,-3\} & 0 & \{0,0\} & 0 \\
\{1,-3\} & 0 & \{0,0\} & 0
\end{array}\right) .
$$

Now let $R^{\prime}=\mathbb{Q}\left[x_{1}, x_{2}, x_{3}, x_{4}\right]$. The cohomology of the complex described by this matrix is the tensor product of the quotient $R^{\prime} /\left(\left(x_{2}-x_{4}\right)\left(x_{4}-x_{1}\right)\right)$ and the bigraded vector space

$$
(\mathbb{Q}\{1,-3\} \oplus \mathbb{Q}) \otimes(\mathbb{Q}\{1,-3\} \oplus \mathbb{Q}) .
$$

The bigraded dimension of $R^{\prime} /\left(\left(x_{2}-x_{4}\right)\left(x_{4}-x_{1}\right)\right)$ has the form $1+\alpha$ where $\alpha \in q^{2} \mathbb{Z}\left[q^{2}\right]$, while that of the second term is $\left(1+t q^{-3}\right)^{2}$. Therefore, the bigraded dimension of the cohomology of the complex $C\left(\Gamma_{110}\right) \bullet \otimes C\left(\Gamma_{100}\right)$ has the form

$$
(1+\alpha)\left(1+2 t q^{-3}+t^{2} q^{-6}\right)
$$

Writing it as a polynomial in $t$ with coefficients being power series in $q$, we see that the coefficient of the term $t^{0} q^{0}$ equals 1 . Therefore, the bidegree $(0,0)$ summand of the homology is one-dimensional, and the hom space $\operatorname{Hom}_{h m f_{w}}\left(C\left(\Gamma_{110}\right), C\left(\Gamma_{100}\right)\right)$ has dimension 1 .

To show that $\chi_{1}: C\left(\Gamma_{110}\right) \longrightarrow C\left(\Gamma_{100}\right)$ (corresponding to the splitting of the right wide edge of $\Gamma_{110}$ into two parallel lines) generates this one-dimensional space, it suffices to show that $\chi_{1}$ is not null-homotopic. We can write the factorizations and the map in the following Koszul form

$$
\left(\begin{array}{cc}
a & x_{1}+x_{2}-x_{4}-x_{7} \\
0 & \left(x_{2}-x_{4}\right)\left(x_{2}-x_{7}\right) \\
a & x_{7}+x_{3}-x_{5}-x_{6} \\
0 & \left(x_{3}-x_{5}\right)\left(x_{3}-x_{6}\right)
\end{array}\right) \stackrel{\chi_{1}}{\longrightarrow}\left(\begin{array}{cc}
a & x_{1}+x_{2}-x_{4}-x_{7} \\
0 & \left(x_{2}-x_{4}\right)\left(x_{2}-x_{7}\right) \\
a & x_{7}+x_{3}-x_{5}-x_{6} \\
0 & x_{3}-x_{6}
\end{array}\right)
$$


with $\chi_{1}=\mathrm{Id}^{\otimes 3} \otimes \psi\left(x_{3}-x_{5}\right)$. Applying row transformation [13] $]_{1}$ to each matrix and then excluding $x_{7}$ we reduce the map to

$$
\left(\begin{array}{cc}
a & x_{1}+x_{2}+x_{3}-x_{4}-x_{5}-x_{6} \\
0 & \left(x_{2}-x_{4}\right)\left(x_{2}+x_{3}-x_{5}-x_{6}\right) \\
0 & \left(x_{3}-x_{5}\right)\left(x_{3}-x_{6}\right)
\end{array}\right) \stackrel{\chi_{1}}{\longrightarrow}\left(\begin{array}{cc}
a & x_{1}+x_{2}+x_{3}-x_{4}-x_{5}-x_{6} \\
0 & \left(x_{2}-x_{4}\right)\left(x_{2}+x_{3}-x_{5}-x_{6}\right) \\
0 & x_{3}-x_{6}
\end{array}\right)
$$

with $\chi_{1}=\mathrm{Id}^{\otimes 2} \otimes \psi\left(x_{3}-x_{5}\right)$. Turn both $\Gamma_{110}$ and $\Gamma_{100}$ into closed diagrams $\widehat{\Gamma}_{110}$ and $\widehat{\Gamma}_{100}$ by connecting top endpoints of each diagram with its bottom endpoints by three disjoint arcs. To check that $\chi_{1}$ is not null-homotopic, it is enough to verify that the induced map on cohomology

$$
\widehat{\chi}_{1}: H\left(\widehat{\Gamma}_{110}\right) \longrightarrow H\left(\widehat{\Gamma}_{100}\right)
$$

is non-trivial. We represent this map in Koszul form as

$$
\left(\begin{array}{cc}
a & x_{1}+x_{2}+x_{3}-x_{4}-x_{5}-x_{6} \\
0 & \left(x_{2}-x_{4}\right)\left(x_{2}+x_{3}-x_{5}-x_{6}\right) \\
0 & \left(x_{3}-x_{5}\right)\left(x_{3}-x_{6}\right) \\
a & x_{4}-x_{1} \\
a & x_{5}-x_{2} \\
a & x_{6}-x_{3}
\end{array}\right) \stackrel{\widehat{x}_{1}}{\longrightarrow}\left(\begin{array}{cc}
a & x_{1}+x_{2}+x_{3}-x_{4}-x_{5}-x_{6} \\
0 & \left(x_{2}-x_{4}\right)\left(x_{2}+x_{3}-x_{5}-x_{6}\right) \\
0 & x_{3}-x_{6} \\
a & x_{4}-x_{1} \\
a & x_{5}-x_{2} \\
a & x_{6}-x_{3}
\end{array}\right),
$$

and $\widehat{\chi}_{1}=\mathrm{Id}^{\otimes 2} \otimes \psi\left(x_{3}-x_{5}\right) \otimes \mathrm{Id}^{\otimes 3}$. Doing transformations $[14]_{1},[15]_{1},[16]_{1}$ and excluding $x_{4}, x_{5}, x_{6}$ and $a$, we reduce the map to the form

$$
\left(\begin{array}{ll}
0 & 0 \\
0 & 0
\end{array}\right) \stackrel{\widehat{\chi}_{1}}{\longrightarrow}\left(\begin{array}{ll}
0 & 0 \\
0 & 0
\end{array}\right),
$$

where $\widehat{\chi}_{1}=\operatorname{Id} \otimes \psi\left(x_{3}-x_{2}\right)$ and the ground ring is $\mathbb{Q}\left[x_{1}, x_{2}, x_{3}\right]$ (we took the quotient by the ideal $\left.\left(x_{1}-x_{4}, x_{2}-x_{5}, x_{3}-x_{6}, a\right)\right)$. Clearly, $\widehat{\chi}_{1}$ induces a nontrivial map on cohomology, and $\chi_{1}$ is not null-homotopic.

Using symmetries of the graphs and factorizations, the other cases of the lemma can be reduced to verifying that the hom spaces

$$
\operatorname{Hom}_{h m f_{w}}\left(\Upsilon, C\left(\Gamma_{110}\right)\right) \text { and } \operatorname{Hom}_{h m f_{w}}\left(C\left(\Gamma_{100}\right), C\left(\Gamma_{000}\right)\right)
$$

are both one-dimensional and generated by $\chi_{1}$. Actual computations, similar to the one above, are left to a curious reader. For the first of the two hom spaces, by $\chi_{1}$ we mean the composition of $\chi_{1}: C\left(\Gamma_{111}\right) \longrightarrow C\left(\Gamma_{110}\right)$ with the inclusion of $\Upsilon$ as a direct summand of $C\left(\Gamma_{111}\right)$. 
Lemma 6 For any arrow $\Gamma \longrightarrow \Gamma^{\prime}$ in Figure 19, factorizations $C(\Gamma)$ and $C\left(\Gamma^{\prime}\right)$ are not isomorphic in $h m f_{w}$.

Proof (Sketch) Form the closures $\widehat{\Gamma}$ and $\widehat{\Gamma}^{\prime}$ by connecting top endpoints of each diagram with its bottom endpoints by 3 disjoint arcs. A direct computation shows that complexes $C(\widehat{\Gamma})$ and $C\left(\widehat{\Gamma}^{\prime}\right)$ have non-isomorphic cohomology groups (their two-variable Poincare polynomials are different).

Thus, the complex $C^{\prime}$, depicted in Figure 19, consists of 6 factorizations and its differential is a sum of 10 maps, one for each arrow of the figure. Each map is either 0 or a nonzero multiple of the unique (up to rescaling) nontrivial map between the two factorizations. For each arrow $b: \Gamma \longrightarrow \Gamma^{\prime}$ choose a nontrivial map $m(b): C(\Gamma) \rightarrow$ $C\left(\Gamma^{\prime}\right)$.

Lemma 7 For any two composable arrows $\Gamma \stackrel{b_{1}}{\longrightarrow} \Gamma^{\prime} \stackrel{b_{2}}{\longrightarrow} \Gamma^{\prime \prime}$ the composition $m\left(b_{2}\right) m\left(b_{1}\right)$ is nontrivial in $h m f_{w}$.

Proof It suffices to check that the composition

$$
\Upsilon \subset C\left(\Gamma_{111}\right) \stackrel{\chi_{1}}{\longrightarrow} C\left(\Gamma_{110}\right) \stackrel{\chi_{1}}{\longrightarrow} C\left(\Gamma_{100}\right) \stackrel{\chi_{1}}{\longrightarrow} C\left(\Gamma_{000}\right)
$$

is not null-homotopic. Denote the composition of the last 3 maps by $\chi_{1}^{\prime}$ and the corresponding "adjoint" composition

$$
C\left(\Gamma_{000}\right) \stackrel{\chi_{0}}{\longrightarrow} C\left(\Gamma_{100}\right) \stackrel{\chi_{0}}{\longrightarrow} C\left(\Gamma_{110}\right) \stackrel{\chi_{0}}{\longrightarrow} C\left(\Gamma_{111}\right)
$$

by $\chi_{0}^{\prime}$. We claim that the map $\chi_{1}^{\prime} p r \chi_{0}^{\prime}$ is non-zero, where pr is the projection from $C\left(\Gamma_{111}\right)$ onto its direct summand $\Upsilon$. The map $\chi_{0}^{\prime}$ has degree $(0,6)$ and the product $\chi_{1}^{\prime} \chi_{0}^{\prime}$ is equal to the multiplication by $\left(x_{4}-x_{2}\right)^{2}\left(x_{5}-x_{3}\right)$ endomorphism of $C\left(\Gamma_{000}\right)$, since the composition $\chi_{1} \chi_{0}$ is the multiplication by a suitable linear combination of the $x$. The complementary direct summand of $C\left(\Gamma_{111}\right)$ is isomorphic to $C\left(\Gamma_{100}\right)\{0,2\}$. Denote by $\widetilde{p r}$ the projection onto this direct summand. Then $\mathrm{pr}+\widetilde{\mathrm{pr}}$ is the identity endomorphism of $C\left(\Gamma_{111}\right)$.

The composition $\chi_{1}^{\prime} \widetilde{\operatorname{pr}} \chi_{0}^{\prime}$ factors though a degree $(0,2)$ endomorphism of $C\left(\Gamma_{110}\right)$. This endomorphism is a composition

$$
C\left(\Gamma_{110}\right) \longrightarrow C\left(\Gamma_{100}\right) \longrightarrow C\left(\Gamma_{110}\right)
$$

where the first map has degree $(0,0)$ and the second-degree $(0,2)$. These maps are, necessarily, rational multiples of $\chi_{0}$ and $\chi_{1}$ (corresponding to the right wide edge of $\Gamma_{110}$ ) and their composition is a rational multiple of the multiplication by 
$x_{3}-x_{5}$. Hence, the composition $\chi_{1}^{\prime} \widetilde{\operatorname{pr}} \chi_{0}^{\prime}$ is a rational multiple of the multiplication by $\left(x_{3}-x_{5}\right)^{2}\left(x_{2}-x_{4}\right)$. To show that

$$
\chi_{1}^{\prime} \operatorname{pr} \chi_{0}^{\prime}=\chi_{1}^{\prime} \chi_{0}^{\prime}-\chi_{1}^{\prime} \widetilde{\operatorname{pr}} \chi_{0}^{\prime}
$$

is not null-homotopic, we observe that the right hand side is the multiplication by

$$
\left(x_{4}-x_{2}\right)^{2}\left(x_{5}-x_{3}\right)-\mu\left(x_{3}-x_{5}\right)^{2}\left(x_{2}-x_{4}\right)
$$

endomorphism of $C\left(\Gamma_{000}\right)$, for some rational $\mu$. The image of $\mathbb{Q}\left[x_{1}, \ldots, x_{6}\right]$ in the endomorphism ring of $C\left(\Gamma_{000}\right)$ is the quotient ring by relations $x_{1}=x_{4}, x_{2}=x_{5}$ and $x_{3}=x_{6}$. The polynomial above simplifies to

$$
\left(x_{1}-x_{2}\right)^{2}\left(x_{2}-x_{3}\right)-\mu\left(x_{3}-x_{2}\right)^{2}\left(x_{2}-x_{1}\right) \neq 0
$$

in $\mathbb{Q}\left[x_{1}, x_{2}, x_{3}\right]$. Therefore, the composition $\chi_{1}^{\prime} \operatorname{pr} \chi_{0}^{\prime}$ is not null-homotopic, and so is the map $\Upsilon \longrightarrow C\left(\Gamma_{000}\right)$ in formula (26). Lemma 7 follows.

The differential in the complex $C^{\prime}$ can be written as

$$
d=\sum_{b} \lambda_{b} m(b),
$$

with $\lambda_{b} \in \mathbb{Q}$, and the sum over all arrows $b$.

Lemma 8 All coefficients $\lambda_{b}$ are nonzero rational numbers.

Proof Assume otherwise: $\lambda_{b}=0$ for some $b$. Every square in the diagram of $C^{\prime}$ anticommutes, and from Lemma 7 we derive that some other $\lambda$ would have to be zero. In fact, there will be enough zero maps to split the complex into the direct sum of at least two subcomplexes, each comprised of two or four factorizations in Figure 19. Specifically, the complex will either decompose into a direct sum of 3 subcomplexes of the form

$$
0 \longrightarrow C(\Gamma) \stackrel{m(b)}{\longrightarrow} C\left(\Gamma^{\prime}\right) \longrightarrow 0
$$

for some three arrows $b$, or as the direct sum of one subcomplex of type (27) and the complementary summand containing the other four factorizations.

A decomposition of $C^{\prime}$ into a direct sum of 3 subcomplexes contradicts Lemma 4 and Lemma 6. To see the impossibility of the decomposition of the second kind, it is enough to show that the complementary summand cannot be trivial in $h m f_{w}$. This summand would consist of four factorizations that sit in the vertices of one of the four squares in Figure 19. For instance, it could have the form

$$
0 \longrightarrow C(Y) \longrightarrow C\left(\Gamma_{110}\right) \oplus C\left(\Gamma_{011}\right) \longrightarrow C\left(\Gamma_{010}\right) \longrightarrow 0 .
$$


Triviality of the summand would imply that its identity map is null-homotopic. In particular, the identity map of the rightmost factorization in the complex would factor through a map to the middle term. This map should have bidegree $(0,0)$. The following lemma establishes the contradiction.

Lemma 9 For any arrow $\Gamma \longrightarrow \Gamma^{\prime}$ in Figure 19 we have

$$
\operatorname{Hom}_{h m f_{w}}\left(C\left(\Gamma^{\prime}\right), C(\Gamma)\right)=0 .
$$

Proof Thus, any bidegree zero map is trivial. The lemma can be proved in the same way as Lemma 5.

Lemma 8 follows.

To summarize, we established that the coefficients $\lambda_{b}$ in the differential for the complex $C^{\prime}$ are all nonzero. Rescaling, if necessary, we can turn them into 1 and -1 . Moreover, the complex $C^{\prime}$ is uniquely determined, up to isomorphism, by the condition that $\lambda_{b} \neq 0$ for all $b$. We have $C\left(D_{1}\right) \cong C^{\prime}$. Nearly identical arguments show that $C\left(D_{2}\right) \cong C^{\prime}$ as well. Therefore, $C\left(D_{1}\right) \cong C\left(D_{2}\right)$, and Proposition 8 follows.

\subsection{Computing the Euler characteristic}

For a braid diagram $D$ consider three more diagrams: the diagrams $D \sigma_{i}$ and $D \sigma_{i}$ resulting from placing a positive or a negative crossing between the $i$ th and ( $i+$ 1) th strands on top of $D$ and the diagram $D e_{i}$ resulting from placing a wide edge between these strands on top of $D$. Equations (12) and (13) (see also Figure 6) present categorification complexes $\widetilde{C H}\left(D \sigma_{i}\right)$ and $\widetilde{C H}\left(D \sigma_{i}^{-1}\right)$ of the braid closures $D \sigma_{i}$, $D \sigma_{i}^{-1}$ as cones of morphisms between the complexes $\widetilde{C H}(D)$ and $\widetilde{C H}\left(D e_{i}\right)$ of the closures of $D$ and $D e_{i}$ :

$$
\begin{aligned}
\widetilde{C H}\left(D \sigma_{i}\right) & =\text { Cone }\left(\widetilde{C H}(D)\left\{-\frac{1}{2}, \frac{3}{2}\right\}\left[-\frac{1}{2}\right] \stackrel{\chi_{0}}{\longrightarrow} \widetilde{C H}\left(D e_{i}\right)\left\{-\frac{1}{2},-\frac{1}{2}\right\}\left[-\frac{1}{2}\right]\right) \\
\widetilde{C H}\left(D \sigma_{i}^{-1}\right) & =\operatorname{Cone}\left(\widetilde{C H}\left(D e_{i}\right)\left\{\frac{1}{2},-\frac{3}{2}\right\}\left[-\frac{1}{2}\right] \stackrel{\chi_{1}}{\longrightarrow} \widetilde{C H}(D)\left\{\frac{1}{2},-\frac{3}{2}\right\}\left[-\frac{1}{2}\right]\right) .
\end{aligned}
$$

As a result, their graded Euler characteristics defined by equation (2) are related as

$$
\begin{aligned}
\left\langle D \sigma_{i}\right\rangle & =-t^{-1} q\langle D\rangle+t^{-1} q^{-1}\left\langle D e_{i}\right\rangle \\
\left\langle D \sigma_{i}^{-1}\right\rangle & =t q^{-1}\left\langle D e_{i}\right\rangle-t q^{-1}\langle D\rangle,
\end{aligned}
$$

so the Euler characteristic $\langle D\rangle$ satisfies the skein relation (4):

$$
t\left\langle D \sigma_{i}\right\rangle-t^{-1}\left\langle D \sigma_{i}^{-1}\right\rangle=-\left(q-q^{-1}\right)\langle D\rangle .
$$


Proof (Theorem 2) If $D$ is a one-strand braid diagram of the unknot then

$$
H(D) \cong \mathbb{Q}[x]\left\{-\frac{1}{2}, \frac{3}{2}\right\}\left[\frac{1}{2}\right]
$$

and

$$
\langle D\rangle=\frac{t^{-1}}{q-q^{-1}} .
$$

Since the HOMFLYPT function is determined uniquely by the skein relation and by its value on the unknot, a comparison between the formulas (30) and (5) leads to the relation (3), which is our normalization of $F(D)$. Propositions proved above imply that $\langle D\rangle$ satisfies all other defining properties of $F(D)$. Theorem 2 follows.

Acknowledgements The idea to consider matrix factorizations with the potential $a x$ came up when the authors were attending Virginia Topology Conference in December of 2004. We would like to thank Slava Krushkal and Frank Quinn, the conference organizers, for bringing us together and creating an inspiring research atmosphere. M K is grateful to Sergei Gukov, Peter Ozsváth, Jacob Rasmussen, Albert Schwarz and Oleg Viro for interesting discussions. We are happy to acknowledge NSF support via grants DMS-0407784 and DMS-0196131.

\section{References}

[1] M M Asaeda, J H Przytycki, A S Sikora, Categorification of the Kauffman bracket skein module of I-bundles over surfaces, Algebr. Geom. Topol. 4 (2004) 1177-1210 MR2113902

[2] B Audoux, T Fiedler, A Jones polynomial for braid-like isotopies of oriented links and its categorification, Algebr. Geom. Topol. 5 (2005) 1535-1553 MR2186108

[3] D Bar-Natan, Khovanov's homology for tangles and cobordisms, Geom. Topol. 9 (2005) 1443-1499 MR2174270

[4] P Freyd, D Yetter, J Hoste, W B R Lickorish, K Millett, A Ocneanu, A new polynomial invariant of knots and links, Bull. Amer. Math. Soc. (N.S.) 12 (1985) 239-246 MR776477

[5] B Gornik, Note on Khovanov link cohomology arXiv:math.QA/0402266

[6] S Gukov, A Schwarz, C Vafa, Khovanov-Rozansky homology and topological strings, Lett. Math. Phys. 74 (2005) 53-74 MR2193547

[7] M Khovanov, Link homology and Frobenius extensions, Fund. Math. 190 (2006) 179-190 MR2232858

[8] M Khovanov, L Rozansky, Matrix factorizations and link homology, Fundamenta Mathematicae 199 (2008) 1-91 
[9] E S Lee, An endomorphism of the Khovanov invariant, Adv. Math. 197 (2005) 554-586 MR2173845

[10] P Ozsváth, Z Szabó, Holomorphic disks and knot invariants, Adv. Math. 186 (2004) 58-116 MR2065507

[11] J H Przytycki, P Traczyk, Conway algebras and skein equivalence of links, Proc. Amer. Math. Soc. 100 (1987) 744-748 MR894448

[12] J Rasmussen, Floer homology and knot complements arXiv:math.GT/0306378

[13] J Rasmussen, Khovanov homology and the slice genus arXiv:math.GT/0402131 to appear in Inventiones Mathematicae

[14] O Viro, Private communication

[15] H Wu, Braids, transversal links and the Khovanov-Rozansky theory arXiv: math.GT/0508064 to appear in Transactions of AMS

Department of Mathematics, Columbia University,

New York, NY 10027

Department of Mathematics, University of North Carolina,

Chapel Hill, NC 27599

khovanov@math.columbia.edu, rozansky@math.unc.edu

Proposed: Peter Ozsvath

Received: 2 February 2006

Seconded: Vaughan Jones, Peter Teichner

Revised: 2 April 2008

Geometry 8 Topology, Volume 12 (2008) 